\title{
FAST SINGULAR OSCILLATING LIMITS AND GLOBAL REGULARITY FOR THE 3D PRIMITIVE EQUATIONS OF GEOPHYSICS *
}

\author{
Anatoli Babin $^{1}$, Alex Mahalov ${ }^{2}$ and Basil Nicolaenko ${ }^{2}$
}

\begin{abstract}
Fast singular oscillating limits of the three-dimensional "primitive" equations of geophysical fluid flows are analyzed. We prove existence on infinite time intervals of regular solutions to the 3D "primitive" Navier-Stokes equations for strong stratification (large stratification parameter $N$ ). This uniform existence is proven for periodic or stress-free boundary conditions for all domain aspect ratios, including the case of three wave resonances which yield nonlinear " $2 \frac{1}{2}$ dimensional" limit equations for $N \rightarrow+\infty$; smoothness assumptions are the same as for local existence theorems, that is initial data in $H_{\alpha}, \alpha \geq 3 / 4$. The global existence is proven using techniques of the Littlewood-Paley dyadic decomposition. Infinite time regularity for solutions of the 3D "primitive" Navier-Stokes equations is obtained by bootstrapping from global regularity of the limit resonant equations and convergence theorems.
\end{abstract}

Mathematics Subject Classification. 76D05, 76D50, 76U05, 86A10.

Received: November 15, 1999

\section{INTRODUCTION}

The governing flow equations for three-dimensional stably stratified fluids with effects of rotation included (the 3D "primitive" equations of geophysics) are under the Boussinesq approximation:

$$
\begin{gathered}
\partial_{t} \mathbf{U}+\mathbf{U} \cdot \nabla \mathbf{U}+f e_{3} \times \mathbf{U}=-\nabla p+\rho_{1} e_{3}+\nu_{1} \Delta \mathbf{U}+\mathbf{F}, \nabla \cdot \mathbf{U}=0, \\
\partial_{t} \rho_{1}+\mathbf{U} \cdot \nabla \rho_{1}=-N^{2} U_{3}+\nu_{2} \Delta \rho_{1}+F_{4}, \\
\left.\mathbf{U}(t, x)\right|_{t=0}=\mathbf{U}(0, x),\left.\rho_{1}(t, x)\right|_{t=0}=\rho_{1}(0, x)
\end{gathered}
$$

where the mean stratification gradient and the axis of rotation are aligned parallel to the vertical axis $e_{3}$. Here $x=\left(x_{1}, x_{2}, x_{3}\right), \mathbf{U}=\left(U_{1}, U_{2}, U_{3}\right)$ is the velocity field and $\rho_{1}$ is the buoyancy variable (relative density variation); $N$ is the Brunt-Väisälä wave frequency for constant stratification and $\Omega$ is the frequency of background rotation, $f=2 \Omega$ is the Coriolis parameter, $\mathbf{F}=\left(F_{1}, F_{2}, F_{3}\right)$ is a divergence free force and $p$ is the pressure. In

Keywords and phrases. Fast singular oscillating limits, three-dimensional Navier-Stokes equations, primitive equations for geophysical fluid flows.

* Dedicated to Roger Temam for his 60th birthday

1 Department of Mathematics, University of California, Irvine, CA, 92697, USA.

2 Department of Mathematics, Arizona State University, Tempe, AZ 85287, USA. 
equations (1.1) $e_{3} \times \mathbf{U}=\left(-U_{2}, U_{1}, 0\right)$ is the Coriolis term. Equations (1.1) and (1.2) are called the primitive (non-hydrostatic) equations of geophysical flows. We consider initial value problem for equations (1.1-1.2) with $\nu_{1}>0$ and $\nu_{2}>0$; here $\nu_{1}$ and $\nu_{2}$ are the kinematic viscosity and the heat conductivity, respectively; the ratio $\operatorname{Pr}=\nu_{1} / \nu_{2}$ is known as the Prandtl number. We consider periodic boundary conditions in a parallepiped $\left[0,2 \pi a_{1}\right] \times\left[0,2 \pi a_{2}\right] \times\left[0,2 \pi a_{3}\right]$, as well as stress-free conditions $U_{3}=0, \partial U_{1} / \partial x_{3}=\partial U_{2} / \partial x_{3}=0$ at $x_{3}=0$, $2 \pi a_{3}$. For stress-free conditions one only needs to restrict Fourier series to be even in $x_{3}$ for $U_{1}, U_{2}$ and odd in $x_{3}$ for $U_{3}, \rho_{1}$ (see $\left.[25]\right)$.

In this paper we investigate the fast singular oscillating limits of equations (1.1-1.2) as $f \rightarrow \infty, N \rightarrow \infty$, $\eta=f / N$ fixed. We average equations (1.1-1.2) over the fast time scales of inertio-gravity waves. The linear parts of inviscid equations (1.1-1.2) are:

$$
\begin{gathered}
\partial_{t} \mathbf{U}+f e_{3} \times \mathbf{U}-\rho_{1} e_{3}=-\nabla p, \quad \nabla \cdot \mathbf{U}=0, \\
\partial_{t} \rho_{1}+N^{2} U_{3}=0 .
\end{gathered}
$$

In our approach, the collective contribution to the nonlinear dynamics made by fast "inertio-gravity" waves (solutions to Eqs. (1.4-1.5)) is accounted for by rigorous estimates of wave resonances and quasi-resonances via small divisors analysis $[4,6,8,12,14,15]$. The linearized equations (1.4) with $f \neq 0, N=0, \rho_{1}=0$ was studied by Sobolev [47] who continued the analysis of Poincaré [43] (Arnold and Khesin [2]). The extension of this analysis to the genuine nonlinear equations (1.1-1.2) was done by Babin, Mahalov and Nicolaenko (henceforth BMN) in $[4-6,8,12,14]$. Utilizing methods of small denominators and Diophantine incommensurability conditions on the domain geometrical parameters $a_{1}, a_{2}, a_{3}$, they investigate the fast singular oscillating limits of equations (1.1), $N=0$ as $f=2 \Omega \rightarrow \infty$. This mathematical approach in the context of geophysical flows was initiated in BMN $[4,6,10,11]$, Mahalov and Marcus [40]. In the context of symmetric hyperbolic systems (including weakly nonlinear geometric optics), related singular oscillating limits have been investigated by Joly, Metivier and Rauch [31,34], Schochet [45] (where the weakly compressible limit of 3D Euler equations was considered), Grenier [30], Embid and Majda [26]. For general "primitive" equations of geophysics, following methods for hyperbolic Euler-type systems of Bardos [17], Bourgeois and Beale [19] have demonstrated convergence to quasi-geostrophic limit equations for special classes of initial data (of infinite codimension). Lions, Temam and Wang have analyzed the viscous equations $(1.1-1.2)$ in a series of seminal papers [37,38], while restricting to "prepared" initial conditions or conditional regularity hypotheses.

First results on regularity of solutions of three-dimensional Euler and Navier-Stokes systems in rapidly rotating frame were obtained in $[5,8]$. First results on regularity in the context of geophysical flows were obtained in $[6,10,11]$. The crucial role of parameters $\theta_{2}=1 / a_{2}^{2}, \theta_{3}=1 / a_{3}^{2}$ for the properties of the dynamics, in particular for smoothness, was revealed in these papers (we put $a_{1}=1$ using a rescaling; in the general case one has to put $\left.\theta_{2}=a_{1}^{2} / a_{2}^{2}, \theta_{3}=a_{1}^{2} / a_{3}^{2}\right)$. Technical conditions of $[5,6]$ and $[8]$ on the smoothness of initial data and forcing term were later relaxed in $[28,29]$ (no consideration of " $2 \frac{1}{2}$-dimensional" nonlinear limit equations from 3 wave resonances) and $[3,12]$. An approach based on choosing special restricted sets of initial data with infinite codimension which do not excite resonant 3-wave interactions is used in [21] to obtain long-time existence theorems.

There are three foremost issues with the analysis of (1.1-1.2) for large parameters $N$ and $f$. First, the nature of the limit asymptotic equations as $N \rightarrow+\infty$ and the regularity of their solutions (" $2 \frac{1}{2}$-dimensional" Navier-Stokes primitive equations). Second, the convergence of solutions of (1.1-1.2) to those of the limit equations; and, finally, bootstrapping from analysis of the first two questions the infinite time regularity of solutions of (1.1-1.2) for $N$ large but finite.

The proof of global regularity of 3D primitive Navier-Stokes equations (1.1-1.2) for resonant domains presented in this paper relies on the global regularity of the " $2 \frac{1}{2}$-dimensional" limit nonlinear "primitive" NavierStokes equations and techniques for convergence theorems as $N \rightarrow \infty$ developed in $[3,6,8,12,14]$. The technique of bootstrapping regularity of solutions of three-dimensional Navier-Stokes equations by perturbation from limit 
equations has been done in various contexts: thin domains [44], helical flows [39]. In these previous works, limit equations are 2-D Navier-Stokes equations for which global regularity is well known. In the present work, the limit equations are genuinely three-dimensional depending on all three variables $x_{1}, x_{2}$ and $x_{3}$ but with restricted wave-number interactions in the nonlinear term. The existence and regularity theory for those limit equations is non-trivial.

In [14], we have demonstrated global regularity of equations $(1.1)$ in the pure rotation case $\left(N=0, \rho_{1}=0\right)$ for large Coriolis parameters $f$ including the case of 3 waves resonances with the " $2 \frac{1}{2}$-dimensional" limit Navier-Stokes equations. In this paper we extend the results of [14] to the full primitive 3D equations (1.1-1.2) $(N \neq 0)$. The main result of this paper is the uniform existence in infinite time of regular strong solutions of equations (1.1-1.2) for large but finite stratification parameters $N$. This result holds for all domain parameters $a_{1}, a_{2}, a_{3}$ including the case of domains with three wave resonances for inertio-gravity waves; such resonances yield strongly nonlinear " $2 \frac{1}{2}$-dimensional" limit equations. The global existence is proven using techniques of the Littlewood-Paley dyadic decomposition.

Smoothness conditions in this paper are like in standard local regularity theorems and do not include technical smoothness conditions of BMN [6,11]. All restrictions on the domain parameters are also removed. We also relax in this paper (in viscous case using the approach of Avrin and BMN [3]) conditions on time behavior of the forcing term. In regularity theorems including Theorems 1.1 and 1.2 we impose only an integral regularity condition on forcing term

$$
\sup _{T} \int_{T}^{T+1}\left\|\mathbf{F}^{\dagger}\right\|_{\alpha-1}^{2} \mathrm{~d} t \leq M_{\alpha F}^{2}
$$

for $\alpha>3 / 4$, where $\mathbf{F}^{\dagger}=\left(\mathbf{F}, F_{4}\right)$. We denote by $\mathbf{U}^{\dagger}$ the 4 -vector $\mathbf{U}^{\dagger}=\left(\mathbf{U}, \rho_{1}\right)$.

In this paper we prove the following main theorems; the Sobolev spaces $H_{\alpha}$ of periodic functions with zero mean are defined in equations $(2.4-2.5)$.

Theorem 1.1. Let $\eta=f / N$ and the domain parameters $a_{1}, a_{2}, a_{3}$ be fixed but arbitrary. Let $\nu_{1}, \nu_{2}>0$, $\nu=\min \left(\nu_{1}, \nu_{2}\right)$ and the condition (1.6) on the force $\mathbf{F}^{\dagger}(t, x)$ be satisfied. Let $\left\|\mathbf{U}^{\dagger}(0)\right\|_{\alpha} \leq M_{\alpha}$ where $\alpha>3 / 4$. Then for $N \geq N_{1}\left(M_{\alpha}, M_{\alpha F}, \nu, a_{1}, a_{2}, a_{3}\right)$ solutions of the 3D Navier-Stokes "primitive" (1.1-1.2) are regular for all $t \geq 0$, and $\left\|\mathbf{U}^{\dagger}(t)\right\|_{\alpha} \leq M_{\alpha}^{\prime}$ for all $t \geq 0$.

Theorem 1.2. Let $\eta=f / N$ and the domain parameters $a_{1}, a_{2}$, a $a_{3}$ be fixed but arbitrary. Let $\nu_{1}, \nu_{2}>0$, $\nu=\min \left(\nu_{1}, \nu_{2}\right), \alpha>3 / 4$ and the condition (1.6) on the force be satisfied. Let $\left\|\mathbf{U}^{\dagger}(0)\right\|_{0} \leq M_{0}, \hat{T}=$ $\hat{T}\left(M_{0}, M_{\alpha F}, \nu\right)$. Then for every $N \geq N^{\prime}\left(a_{1}, a_{2}, a_{3}, \nu, M_{\alpha F}\right), N^{\prime}$ independent of $M_{0}$ and for every weak solution $\mathbf{U}^{\dagger}\left(t, x_{1}, x_{2}, x_{3}\right)$ of the three-dimensional "primitive" Navier-Stokes equations (1.1-1.2) defined on $[0, \hat{T}]$ which satisfies the classical energy estimates on $[0, \hat{T}]$, the following holds: $\mathbf{U}^{\dagger}\left(t, x_{1}, x_{2}, x_{3}\right)$ can be extended to $0<$ $t<+\infty$ and it is regular for every $t: \hat{T} \leq t<+\infty ; \mathbf{U}^{\dagger}\left(t, x_{1}, x_{2}, x_{3}\right)$ belongs to $H_{\alpha}$ and $\left\|\mathbf{U}^{\dagger}\left(t, x_{1}, x_{2}, x_{3}\right)\right\|_{\alpha} \leq$ $C_{1}\left(a_{1}, a_{2}, a_{3}, M_{\alpha F}, \nu\right)$ for every $t \geq \hat{T}$. If $\mathbf{F}^{\dagger}$ is independent of $t$ then there exists a global attractor for the three-dimensional primitive Navier-Stokes equations (1.1-1.2) bounded in $H_{\alpha}$; such an attractor has a finite fractal dimension and attracts every weak Leray solution as $t \rightarrow+\infty$.

In this paper we establish infinite time regularity theorems valid for all domain parameters: for $N$ large but finite in equations $(1.1-1.2)$ this is obtained by bootstrapping from global regularity of the limit " $2 \frac{1}{2}$ dimensional" equations and convergence theorems including the case of domains resonant in $a_{1}, a_{2}, a_{3}$.

Remark 1.1. In [14] only the condition $\alpha>1 / 2$ is imposed on the force and the initial data. Here the condition $\alpha>3 / 4$ is restricted only by the minimal regularity results for the viscous quasi-geostrophic equations, $c f$. Section 4 in [12]. 


\section{The LIMIT RESONANT EQUATIONS}

Following Métais and Herring [41] we introduce a change of variables $\rho_{1}=N \rho$ and combine velocity and buoyancy variable in one variable $\mathbf{U}^{\dagger}=(\mathbf{U}, \rho)$ after which equations (1.1-1.2) written in non-dimensional variables take the more symmetric form:

$$
\begin{array}{r}
\partial_{t} \mathbf{U}^{\dagger}+\mathbf{U} \cdot \nabla \mathbf{U}^{\dagger}+N \mathbf{M} \mathbf{U}^{\dagger}=-\nabla^{\dagger} p+\overline{\boldsymbol{\nu}} \Delta \mathbf{U}^{\dagger}+\mathbf{F}^{\dagger}, \nabla \cdot \mathbf{U}=0 \\
\left.\mathbf{U}^{\dagger}(t, x)\right|_{t=0}=\mathbf{U}^{\dagger}(0, x)
\end{array}
$$

where $\nabla^{\dagger} p=(\nabla p, 0), \mathbf{F}^{\dagger}=\left(\mathbf{F}, F_{4}\right)$ (where $F_{4}$ is rescaled),

$$
\mathbf{M}=(\mathbf{S}+\eta \mathbf{R}), \eta=f / N, \mathbf{R}=\left(\begin{array}{ll}
\mathbf{J} & \mathbf{0} \\
\mathbf{0} & \mathbf{0}
\end{array}\right), \mathbf{S}=\left(\begin{array}{cc}
\mathbf{0} & \mathbf{0} \\
\mathbf{0} & \mathbf{J}
\end{array}\right), \mathbf{J}=\left(\begin{array}{cc}
0 & -1 \\
1 & 0
\end{array}\right),
$$

$\overline{\boldsymbol{\nu}}=\operatorname{diag}\left(\nu_{1}, \nu_{1}, \nu_{1}, \nu_{2}\right)$ is the viscosity matrix, $\eta$ is fixed. The linear parts of inviscid equations $(2.1)$ are:

$$
\partial_{t} \mathbf{U}^{\dagger}+N \mathbf{M} \mathbf{U}^{\dagger}=-\nabla^{\dagger} p, \nabla \cdot \mathbf{U}=0
$$

where the skew-symmetric structure is apparent.

We use Fourier series expansions for redefined fields $\mathbf{U}^{\dagger}(x)=\left(U_{1}(x), U_{2}(x), U_{3}(x), \rho(x)\right), x=\left(x_{1}, x_{2}, x_{3}\right)$ :

$$
\mathbf{U}^{\dagger}(x)=\sum_{n} \exp \left(i\left(n_{1} x_{1}+n_{2} x_{2} / a_{2}+n_{3} x_{3} / a_{3}\right)\right) \mathbf{U}_{n}^{\dagger}=\sum_{n} \exp (i \check{n} \cdot x) \mathbf{U}_{n}^{\dagger}
$$

where $\mathbf{U}_{n}^{\dagger}$ are the (4-component) Fourier coefficients, $\left[n_{1}, n_{2}, n_{3}\right] \in \mathbb{Z}^{3}, \check{n}=\left[n_{1}, n_{2} / a_{2}, n_{3} / a_{3}\right]$ are wavenumbers ( $a_{1}=1$ without loss of generality). We introduce Fourier-Sobolev spaces of functions $H_{s}$ with the norm defined on Fourier coefficients $\mathbf{U}_{n}$ as follows (where $\left.|\check{n}|=\left(n_{1}^{2}+n_{2}^{2} / a_{2}^{2}+n_{3}^{2} / a_{3}^{2}\right)^{1 / 2}\right)$ :

$$
\left\|\mathbf{U}^{\dagger}\right\|_{H_{s}}^{2}=\sum_{n}|\check{n}|^{2 s}\left|\mathbf{U}_{n}^{\dagger}\right|^{2}
$$

We assume that all functions have zero average over the periodic parallepiped. Stress-free boundary conditions at $x_{3}=0,2 \pi a_{3}$ correspond to $U_{1}, U_{2}$ even in $x_{3}$ and $U_{3}, \rho$ odd in $x_{3}$. Sobolev spaces are restricted to such functions. In this paper $\mathbf{R}_{n}, \mathbf{S}_{n}$ will denote the action of $\mathbf{R}$ and $\mathbf{S}$ on $n$-th Fourier component, $\eta \mathbf{R}_{n}+\mathbf{S}_{n}=\mathbf{M}_{n}$.

We recall the principle of averaging equations (2.1) over the fast time scales of inertio-gravity waves. We denote by $\mathbf{E}(N t)$ the linear propagator solution to the initial value problem for equation $(2.3)$; $\mathbf{E}(N t)$ is in fact a unitary group operator (preserves all Sobolev norms). The dispersion relation for inertio-gravity waves which are solutions of equations (1.4-1.5) (hence Eq. (2.3)) has the form

$$
N^{2} \omega_{n}^{2}=N^{2} \frac{\left|\check{\mathrm{n}}^{\prime}\right|^{2}}{|\check{\mathrm{n}}|^{2}}+f^{2} \frac{\check{\mathrm{n}}_{3}^{2}}{|\check{\mathrm{n}}|^{2}}=N^{2}\left(\frac{\left|\check{\mathrm{n}}^{\prime}\right|^{2}}{|\check{\mathrm{n}}|^{2}}+\eta^{2} \frac{\check{\mathrm{n}}_{3}^{2}}{|\check{\mathrm{n}}|^{2}}\right), \omega_{n}^{2}=\frac{\left|\check{\mathrm{n}}^{\prime}\right|^{2}}{|\check{\mathrm{n}}|^{2}}+\eta^{2} \frac{\check{\mathrm{n}}_{3}^{2}}{|\check{\mathrm{n}}|^{2}}
$$

where $\check{\mathrm{n}}=\left(n_{1} / a_{1}, n_{2} / a_{2}, n_{3} / a_{3}\right), \check{\mathrm{n}}^{\prime}=\left(n_{1} / a_{1}, n_{2} / a_{2}, 0\right),|\check{\mathrm{n}}|^{2}=n_{1}^{2} / a_{1}^{2}+n_{2}^{2} / a_{2}^{2}+n_{3}^{2} / a_{3}^{2},\left|\check{n}^{\prime}\right|^{2}=n_{1}^{2} / a_{1}^{2}+n_{2}^{2} / a_{2}^{2}$, $\eta=f / N$. Here $a_{1}, a_{2}$ and $a_{3}$ denote aspect ratios of the domain parallepiped. We note that all results in our work extend to boundary conditions periodic horizontally with zero flux in the vertical direction $e_{3}$ and no tangential stress on the boundary. One only needs to restrict Fourier series to be even in $x_{3}$ for $U_{1}$, $U_{2}$ and odd in $x_{3}$ for $U_{3}, \rho_{1}$. Such boundary conditions imply zero tangential stress on the vertical boundary (see [25]). It follows from (2.6) that the effects of rotation and stratification are not uniform on scales. In the case $\left|\check{n}^{\prime}\right| /\left|\check{n}_{3}\right| \gg 1$ gravity waves are fast and inertial waves are slow. On the other hand, for scales satisfying $\left|\check{n}_{3}\right| /\left|\check{n}^{\prime}\right| \gg 1$ gravity waves are slow and inertial waves are faster. This nonuniformity of the effects of rotation 
and stratification on different scales lies at the very heart of the nonlinear scale adjustment process described in $[10,11]$ and $[15]$.

We introduce the linear propagator directly into the nonlinearity in equation (2.1) using the change of variables

$$
\mathbf{U}^{\dagger}(t)=\mathbf{E}(-N t) \mathbf{u}^{\dagger}(t)
$$

where $\mathbf{U}^{\dagger}=\left(U_{1}, U_{2}, U_{3}, \rho\right)$ is the "fast" field variable; and $\mathbf{u}^{\dagger}$ is the "slow" called Poincaré's variable, after factorization via the fast oscillating $(N \gg 1)$ propagator $\mathbf{E}(N t)$. We define

$$
\mathbf{B}\left(\mathbf{U}^{\dagger}, \mathbf{U}^{\dagger}\right)=\left(-\mathbf{P}(\mathbf{U} \cdot \nabla \mathbf{U}),-\mathbf{U} \cdot \nabla U_{4}\right), \mathbf{U}^{\dagger}=\left(\mathbf{U}, U_{4}\right)=(\mathbf{U}, \rho)
$$

where $\mathbf{P}$ is the Leray projection on divergence free vector fields. For $\nu_{1}=\nu_{2}=0$ and $\mathbf{F}^{\dagger}=0$ the rescaled "primitive" Euler-Boussinesq equations (2.1) written in the Poincaré's variables $\mathbf{u}^{\dagger}$ have the form:

$$
\partial_{t} \mathbf{u}^{\dagger}=\mathbf{B}_{p}\left(N t, \mathbf{u}^{\dagger}, \mathbf{u}^{\dagger}\right), \mathbf{B}_{p}\left(N t, \mathbf{u}^{\dagger}, \mathbf{u}^{\dagger}\right)=\mathbf{E}(N t) \mathbf{B}\left(\mathbf{E}(-N t) \mathbf{u}^{\dagger}, \mathbf{E}(-N t) \mathbf{u}^{\dagger}\right)
$$

where $\mathbf{B}_{p}$ is now an explicitly fast oscillating, non-autonomous operator in the "slow" variable $\mathbf{u}^{\dagger}$. Equation (2.9) are explicitly time-dependent with rapidly varying coefficients. This is a problem of fast singular oscillating limits for a non-local hyperbolic system. Analogous problems are found in nonlinear geometric optics, [31,34].

The following equations describing the asymptotic limit dynamics are associated with equation (2.9) $(\mathrm{BMN},[6])$ :

$$
\partial_{t} \mathbf{w}=\tilde{\mathbf{B}}(\mathbf{w}, \mathbf{w}), \tilde{\mathbf{B}}(\mathbf{w}, \mathbf{w})=\lim _{N \rightarrow+\infty} \frac{1}{2 \pi} \int_{0}^{2 \pi} \mathbf{B}_{p}(N s, \mathbf{w}, \mathbf{w}) \mathrm{d} s
$$

where the arguments $\mathbf{w}$ are $s$-independent. We refer to Lemma 4.1 for the convergence proof. Equation (2.10) contains the " $2 \frac{1}{2}$-dimensional" limit resonant equations. Clearly, when represented in Fourier modes in the limit $N \rightarrow+\infty, \eta=f / N$ fixed, the right-hand side of (2.10) will be determined by resonances $\pm \omega_{k}^{\prime} \pm \omega_{m}^{\prime} \pm \omega_{n}^{\prime}=0$ within terms of the type $\exp \left(i N\left( \pm \omega_{k}^{\prime} \pm \omega_{m}^{\prime} \pm \omega_{n}^{\prime}\right) t\right.$, equation (2.6). Here $\omega_{n}^{\prime}=0$ for quasi-geostrophic (QG) modes and $\omega_{n}^{\prime}=\omega_{n}$ is given by (2.6) for ageostrophic (AG) modes (similarly, $\omega_{k}$ and $\omega_{m}$ ). With $\omega_{n}$ being the normalized spectral frequencies of inertio-gravity waves given by (2.6), the dependence of resonances

$$
D_{l}(k, m, n)= \pm \omega_{k}^{\prime}\left(a_{1}, a_{2}, a_{3}, \eta\right) \pm \omega_{m}^{\prime}\left(a_{1}, a_{2}, a_{3}, \eta\right) \pm \omega_{n}^{\prime}\left(a_{1}, a_{2}, a_{3}, \eta\right)=0
$$

where $l=1, \ldots, 8$ (eight combinations of,+ - signs) and quasi-resonances

$$
D_{l}(k, m, n)= \pm \omega_{k}^{\prime}\left(a_{1}, a_{2}, a_{3}, \eta\right) \pm \omega_{m}^{\prime}\left(a_{1}, a_{2}, a_{3}, \eta\right) \pm \omega_{n}^{\prime}\left(a_{1}, a_{2}, a_{3}, \eta\right)=\delta
$$

on the parameters of the problem $a_{1}, a_{2}, a_{3}$ and $\eta$ and the algebraic geometry of this non-standard small divisor problem are the basis of our analysis for $3 \mathrm{D}$ "primitive" equations in $[6,8,12,14]$. In equation $(2.12) \delta=0$ for exact resonances and is a small parameter for quasi-resonances (see [1]).

Now we describe the structure of the limit equations which were derived in $[4,6,10,11]$. From now on we are going to restrict ourselves to $\eta$ bounded, $\eta>0$, including $\eta \ll 1$. The case of strong rotation and weak stratification $\eta \gg 1$ must be treated separately and it will be published elsewhere. The case $\eta=\infty(f \rightarrow \infty$, $N=0)$ was the subject of our papers on pure fast rotating limit without stratification $([4,5,7,9,14])$.

For all parameters $a_{1}, a_{2}$ and $a_{3}$ and all values of the parameter $\eta=f / N$ in the asymptotic limit equations the total field splits into the quasi-geostrophic field $\mathbf{w}_{Q G}(t)$ satisfying $3 \mathrm{DQG}$ (quasi-geostrophic) equations [6, $10,11,19]$

$$
\partial_{t} \mathbf{w}_{Q G}=\mathbf{B}_{0}\left(\mathbf{w}_{Q G}, \mathbf{w}_{Q G}\right)+\nu_{Q G} \Delta \mathbf{w}_{Q G}+\mathbf{F}_{Q G},
$$


and the ageostrophic components $\mathbf{w}_{A G}$ satisfying equations of the type:

$$
\partial_{t} \mathbf{w}_{A G}=\mathbf{B}_{2}\left(\mathbf{w}_{Q G}, \mathbf{w}_{A G}\right)+\mathbf{B}_{3}\left(\mathbf{w}_{A G}, \mathbf{w}_{A G}\right)+\nu_{A G} \Delta \mathbf{w}_{A G}+\mathbf{F}_{A G}
$$

here $\nu_{Q G}\left(\nu_{1}, \nu_{2}\right)$ and $\nu_{A G}\left(\nu_{1}, \nu_{2}\right)$ are in general non local zeroth-order pseudodifferential operators, whenever $\nu_{1} \neq \nu_{2}$. Equation (2.14) are called the limit resonant " $2 \frac{1}{2}$ dimensional" primitive Navier-Stokes equation.

The limit (2.13) results from the "slow" ( $\left.\mathbf{w}_{Q G}, \mathbf{w}_{Q G}, \mathbf{w}_{Q G}\right)$ triads as well as all resonant

$\left(\mathbf{w}_{A G}, \mathbf{w}_{A G}, \mathbf{w}_{Q G}\right)$ triads (the contribution of the latter is exactly zero in the limit, hence the operator splitting). The limit ageostrophic (2.14) is derived from both resonant $\left(\mathbf{w}_{A G}, \mathbf{w}_{Q G}, \mathbf{w}_{A G}\right)$ and $\left(\mathbf{w}_{Q G}, \mathbf{w}_{A G}, \mathbf{w}_{A G}\right)$ triads as well as the strict 3 -wave resonances $\left(\mathbf{w}_{A G}, \mathbf{w}_{A G}, \mathbf{w}_{A G}\right)$. Notice that the slow-fast-slow $\left(\mathbf{w}_{Q G}, \mathbf{w}_{A G}, \mathbf{w}_{Q G}\right)$ triads are not resonant to the lowest order in $1 / N$ and appear only at the next order in $1 / N$ via 4 -wave resonances (see also [18]).

The quasigeostrophic equations in the inviscid case have a global regular solution according to Bourgeois and Beale [19]; also if $\nu_{1}>0$ according to a theorem proven in [12]. Note that the nonlinear operator $\mathbf{B}_{3}$ discontinuously depends on the parameters $\eta, \theta_{1}=1 / a_{1}^{2}, \theta_{2}=1 / a_{2}^{2}, \theta_{3}=1 / a_{3}^{2}$ (see also Sect. 4); it is non-zero only on a set of measure zero (see the proof in BMN [8] for the similar pure rotating case). In BMN $[5,6,8,10,11]$ it was shown that if one deletes a resonant set $\Theta^{*}$ of parameters $\eta, \theta_{1}, \theta_{2}, \theta_{3}$, then $\mathbf{B}_{3}\left(\mathbf{w}_{A G}, \mathbf{w}_{A G}\right)=0$ and only "catalytic" interactions described by the linear in $\mathbf{w}_{A G}$ operator $\mathbf{B}_{2}\left(\mathbf{w}_{Q G}, \mathbf{w}_{A G}\right)$ rule AG dynamics:

$$
\partial_{t} \mathbf{w}_{A G}=\mathbf{B}_{2}\left(\mathbf{w}_{Q G}, \mathbf{w}_{A G}\right)+\nu_{A G} \Delta \mathbf{w}_{A G}+\mathbf{F}_{A G},
$$

where $\mathbf{w}_{Q G}(t)$ is a solution of $3 \mathrm{DQG}$ equations. In this paper we treat the case of the full " $2 \frac{1}{2}$-dimensional" equations(2.14), including all 3 -waves resonances $\left(\mathbf{B}_{3} \neq 0\right.$ in Eq. (2.14)).

\section{The Limit Resonant EqUATIONS In CRAYA-Fourier BASIS}

In this section we present the limit resonant " $2 \frac{1}{2}$-dimensional" primitive Navier-Stokes equations in CrayaFourier cyclic basis. The Craya basis was originally introduced in Craya [24].

We take into account divergence-free condition by applying the Helmholtz projection $\mathbf{P}^{d}$ onto divergence-free vector fields. The matrix $\left(\mathbf{P}^{d} \mathbf{M} \mathbf{P}^{d}\right)_{n}$ is a real skew-symmetric matrix; the corresponding operator restricted to the 3-dimensional subspace of divergence-free vectors $\mathbf{U}_{n}^{\dagger}$ has one zero eigenvalue and two complex conjugate eigenvalues $\pm i \omega_{n} \neq 0$. We introduce the divergence-free vectors (3.2) which form a real cyclic basis for it:

$$
\mathbf{P}_{n}^{d} \mathbf{M} q_{0 n}=0, \mathbf{P}_{n}^{d} \mathbf{M} q_{1 n}=-\omega_{n} q_{2 n}, \mathbf{P}_{n}^{d} \mathbf{M} q_{2 n}=\omega_{n} q_{1 n},
$$

where $\mathbf{P}_{n}^{d} q_{j n}=q_{j n}$

$$
q_{0 n}=\frac{1}{\omega_{n}}\left(\phi_{n} p_{0 n}+\eta \xi_{n} p_{2 n}\right), q_{1 n}=p_{1 n}, q_{2 n}=\frac{1}{\omega_{n}}\left(\phi_{n} p_{2 n}-\eta \xi_{n} p_{0 n}\right) .
$$

Here $p_{0 n}, p_{1 n}, p_{2 n}$ form an orthonormal basis of the divergence-free subspace for $n$-th Fourier mode; the $p_{j n}$ are the Craya basis for the purely stratified problem [24]:

$$
p_{0 n}=\left[-\frac{\check{n}_{2}}{\left|\check{n}^{\prime}\right|}, \frac{n_{1}}{\left|\check{n}^{\prime}\right|}, 0,0\right], \quad p_{1 n}=\left[\frac{n_{1} \check{n}_{3}}{|\check{n}|\left|\check{n}^{\prime}\right|}, \frac{\check{n}_{2} \check{n}_{3}}{|\check{n}|\left|\check{n}^{\prime}\right|}, \frac{-n_{1}^{2}-\check{n}_{2}^{2}}{|\check{n}|\left|\check{n}^{\prime}\right|}, 0\right], \quad p_{2 n}=e_{4}=[0,0,0,1] .
$$

The eigenvalues $\pm i \omega_{n}$ are given by

$$
\omega_{n}=\sqrt{\phi_{n}^{2}+\eta^{2} \xi_{n}^{2}}, \quad \xi_{n}=\frac{\check{n}_{3}}{|\check{n}|}, \phi_{n}=\frac{\left|\check{n}^{\prime}\right|}{|\check{n}|}, \quad \eta=f / N
$$


where $|\check{n}|^{2}=n_{1}^{2}+n_{2}^{2} / a_{2}^{2}+n_{3}^{2} / a_{3}^{2},\left|\check{n}^{\prime}\right|^{2}=n_{1}^{2}+n_{2}^{2} / a_{2}^{2}$. We consider the case when the ratio $\eta=f / N$ is bounded, by a bounded $\eta_{0}>1$ :

$$
\begin{gathered}
1 / \eta_{0} \leq \eta=f / N \leq \eta_{0}, \\
1 / \eta_{0} \leq \min (1, \eta) \leq \omega_{n} \leq \max (1, \eta) \leq \eta_{0} .
\end{gathered}
$$

In the case $n_{1}=n_{2}=0$ (this case corresponds to taking horizontal averages) we choose the basis which is obtained from (3.2) by putting $n_{1}=n_{2} \neq 0$ and taking $n_{1} \rightarrow 0$. In particular, when $n_{1}=n_{2}=0$ we obtain $\omega_{n}=\eta$ and the eigenvectors are

$$
q_{0 \bar{n}}=(0,0,0,1), q_{1 \bar{n}}=\left(\frac{1}{\sqrt{2}}, \frac{1}{\sqrt{2}}, 0,0\right), q_{2 \bar{n}}=\left(\frac{1}{\sqrt{2}},-\frac{1}{\sqrt{2}}, 0,0\right)
$$

where $\bar{n}=\left(0,0, n_{3}\right)$ denotes wavenumbers for which $n_{1}=n_{2}=0$.

Any arbitrary divergence-free vector field $\mathbf{U}_{n}^{\dagger}$ can be written as

$$
\mathbf{U}_{n}^{\dagger}=V_{n}^{0} q_{0 n}+V_{n}^{1} q_{1 n}+V_{n}^{2} q_{2 n} .
$$

We shall use the variables $V$ to denote vector of coefficients corresponding to $\mathbf{U}_{n}^{\dagger}: \mathbf{V}_{n}=\left[V_{n}^{0}, V_{n}^{1}, V_{n}^{2}\right]=\left[V_{n}^{0}, \mathbf{V}_{n}^{\prime}\right]$, $\mathbf{V}_{n}^{\prime}=\left[V_{n}^{1}, V_{n}^{2}\right]$. Note that the relation between $\mathbf{U}^{\dagger}$ and $\mathbf{V}$ variables is given by

$$
V_{n}^{0}=\mathbf{U}_{n}^{\dagger} \cdot q_{0 n}, V_{n}^{1}=\mathbf{U}_{n}^{\dagger} \cdot q_{1 n}, V_{n}^{2}=\mathbf{U}_{n}^{\dagger} \cdot q_{2 n}
$$

Clearly, $V_{n}^{0^{*}}=-V_{-n}^{0}$ and $V_{n}^{i *}=V_{-n}^{i}, i=1,2$, for real $\mathbf{U}(x)$ and $\rho(x)$. We denote by $\Pi_{n}^{Q G}$ the projection onto $q_{0 n}$ and call it as usual the quasi-geostrophic mode:

$$
\Pi^{Q G} \mathbf{U}^{\dagger}(x)=\sum_{n} V_{n}^{0} q_{0 n} e^{i \check{n} \cdot x}, \Pi_{n}^{Q G} \mathbf{U}_{n}^{\dagger}=V_{n}^{0} q_{0 n} .
$$

The projection onto two-dimensional subspace corresponding to $\pm i \omega_{n}$ is denoted by $\Pi^{A G}$ and defines the ageostrophic component:

$$
\Pi_{n}^{A G} \mathbf{U}_{n}^{\dagger}=V_{n}^{1} q_{1 n}+V_{n}^{2} q_{2 n} .
$$

The case when $\eta \rightarrow 0$ or $\eta \rightarrow \infty$ was discussed in BMN [6]; detailed mathematical consideration of this case can be done along the lines of this paper and BMN [8], but requires additional non-trivial considerations; in particular structure of resonant sets and smoothness conditions are different from those imposed here.

Equation (2.1) in Fourier representation in $V$ variables can be written in the cyclic basis (3.2) as

$$
\partial_{t} V_{n}^{i_{3}}=-i \sum_{k+m=n, i_{1}, i_{2}} Q_{k m n}^{i_{1} i_{2} i_{3}} V_{k}^{i_{1}} V_{m}^{i_{2}}-N \omega_{n}\left(M_{n}^{\prime} V_{n}\right)^{i_{3}}-\left(\hat{\boldsymbol{\nu}}|\check{n}|^{2} \mathbf{V}_{n}\right)^{i_{3}}+F_{n}^{i_{3}}
$$

where $i_{1}, i_{2}, i_{3}=0,1,2, M^{\prime}$ is the matrix $M$ in $V$-variables given by $(3.11) ; \hat{\boldsymbol{\nu}}$ is the viscosity matrix $\overline{\boldsymbol{\nu}}$ in the $V$-basis. Here $\mathbf{J}, \mathbf{M}_{n}^{\prime}$ are given by

$$
\begin{gathered}
\mathbf{M}_{n}^{\prime}=\left(\begin{array}{ccc}
0 & 0 & 0 \\
0 & 0 & -1 \\
0 & 1 & 0
\end{array}\right), \quad \mathbf{J}=\left(\begin{array}{cc}
0 & -1 \\
1 & 0
\end{array}\right), \\
\hat{\boldsymbol{\nu}}=\frac{1}{\omega_{n}^{2}}\left(\begin{array}{ccc}
\nu_{1} \phi_{n}^{2}+\eta^{2} \xi_{n}^{2} \nu_{2} & 0 & \left(\nu_{1}-\nu_{2}\right) \eta \xi_{n} \phi_{n} \\
0 & \nu_{1} \omega_{n}^{2} & 0 \\
\left(\nu_{1}-\nu_{2}\right) \eta \xi_{n} \phi_{n} & 0 & \nu_{2} \phi_{n}^{2}+\eta^{2} \xi_{n}^{2} \nu_{1}
\end{array}\right) .
\end{gathered}
$$


In equation (3.10) quasi-geostrophic modes correspond to 0 eigenvalue of the linear problem and ageostrophic modes are cyclic, see (3.11) and (3.1). The coefficients $Q_{k m n}^{i_{1} i_{2} i_{3}}$ are determined from the equation using (3.2), see BMN [11]:

$$
Q_{k m n}^{i_{1} i_{2} i_{3}}=\left(q_{i_{1} k} \cdot m\right)\left(q_{i_{2} m} \cdot q_{i_{3} n}\right) .
$$

We use notation for the skew-symmetric product $n^{\prime} \wedge m^{\prime} \equiv n_{1} m_{2}-n_{2} m_{1}$. To save space, we give formulas only for 0-wave interactions, see BMN [11] for general coefficients:

$$
Q_{k m n}^{000}=\frac{\omega_{m}|\check{m}| \check{n}^{\prime} \wedge \check{m}^{\prime}}{\omega_{k} \omega_{n}|\check{k}||\check{n}|}
$$

when $n=k+m$; clearly $\left|Q_{k m n}^{i_{1} i_{2} i_{3}}\right| \leq|\check{m}|$.

In equation (3.10)

$$
\partial_{t} \mathbf{V}_{n}+N \omega_{n} \mathbf{M}_{n}^{\prime} \mathbf{V}_{n}=(\mathbf{B}(\mathbf{V}, \mathbf{V}))_{n}-A_{n} \mathbf{V}_{n}+\mathbf{F}_{n}, A_{n}=\hat{\boldsymbol{\nu}}|\check{n}|^{2}
$$

we introduce the linear propagator directly into nonlinearity using the change of variables

$$
\mathbf{V}=\mathbf{E}(-N t) \mathbf{v}, \mathbf{V}_{n}=\exp \left(-N \omega_{n} \mathbf{M}_{n}^{\prime} t\right) \mathbf{v}_{n}
$$

where $\mathbf{v}=\left[v^{0}, v^{1}, v^{2}\right]$ and $\mathbf{M}^{\prime}$ is defined by (3.11).

The action of the linear propagator on the Fourier components $\mathbf{E}(N t)$ can be written in $V$-variables in the Craya cyclic basis using (3.11):

$$
\mathbf{E}(N t)\left[V^{0}, \mathbf{V}^{\prime}\right]_{n}=\exp \left(N \omega_{n} t \mathbf{M}_{n}^{\prime}\right)\left[V^{0}, \mathbf{V}^{\prime}\right]_{n}=\left[V^{0}, \exp \left(N \omega_{n} t \mathbf{J}\right) \mathbf{V}^{\prime}\right] .
$$

Obviously, $\mathbf{E}(N t)$ represents vector rotation in $V^{1}, V^{2}$-plane; orthogonal $V^{0}$ component (called QG) is not affected. To save space, we always write $\mathbf{V}_{n}=\left[V^{0}, \mathbf{V}^{\prime}\right]_{n}$ as a row, understanding that it is a column in the matrix multiplication. Equation (3.15) written in $\mathbf{v}$ variables have the form

$$
\begin{gathered}
\partial_{t} \mathbf{v}=\mathbf{B}(N t, \mathbf{v}, \mathbf{v})-\mathbf{E}(N t) A \mathbf{E}(-N t) \mathbf{v}+\mathbf{F}_{Q G}+E(N t) \mathbf{F}_{A G} \\
\mathbf{B}(N t, \mathbf{v}, \mathbf{v})=\mathbf{E}(N t) \mathbf{B}(\mathbf{E}(-N t) \mathbf{v}, \mathbf{E}(-N t) \mathbf{v})
\end{gathered}
$$

where $\mathbf{F}^{\dagger}=\mathbf{F}_{Q G}+\mathbf{F}_{A G}$ in Craya basis. Equation (3.18) are explicitly time-dependent with rapidly varying coefficients. The corresponding equations for Fourier coefficients have the form:

$$
\begin{array}{r}
\partial_{t} v_{n}^{i_{3}}=\sum_{n=k+m, i_{1}, i_{2} ; \pm \omega_{k}^{\prime} \pm \omega_{m}^{\prime} \pm \omega_{n}^{\prime}=0} \tilde{Q}_{k m n}^{i_{1} i_{2} i_{3}} v_{k}^{i_{1}} v_{m}^{i_{2}}+\sum_{n=k+m, i_{1}, i_{2}} \hat{Q}_{k m n}^{i_{1} i_{2} i_{3}}(N t) v_{k}^{i_{1}} v_{m}^{i_{2}} \\
-\tilde{A}_{n} v_{n}^{i_{3}}-\hat{A}_{n}(N t) v_{n}^{i_{3}}+\mathbf{F}_{Q G, n}+\mathbf{E}_{n}(N t) \mathbf{F}_{A G, n}
\end{array}
$$

where the first sum consists of resonant terms. In the second sum every matrix element $\hat{Q}_{k m n}^{i_{1} i_{2} i_{3}}(N t)$ of nonresonant part as well as $\hat{A}_{n}(N t)$ equals a sum of terms of the form $C \exp \left( \pm i D_{\ell} N t\right)$ with $D_{\ell} \neq 0$. Generally, $D_{\ell}= \pm \omega_{n}^{\prime} \pm \omega_{m}^{\prime} \pm \omega_{k}^{\prime}, \ell=1, \ldots, 8$, where either $\omega_{n}^{\prime}=\omega_{n}$ or $\omega_{n}^{\prime}=0$ and, similarly, for $k$ and $m$. When $D_{\ell}=0$ we call these interactions resonant, when $D_{\ell} \neq 0$ the interactions are non-resonant; see BMN [8] for more details. When all three $\omega_{n}^{\prime}, \omega_{m}^{\prime}, \omega_{k}^{\prime}$ are non-zero we have strict 3-wave resonances; when exactly two of $\omega_{n}^{\prime}, \omega_{m}^{\prime}, \omega_{k}^{\prime}$ are non-zero we have 2-wave resonances; when exactly one of $\omega_{n}^{\prime}, \omega_{m}^{\prime}, \omega_{k}^{\prime}$ is non-zero we have 1-wave resonances. 
The resonant contribution $\tilde{A}_{n}$ from the viscous term does not coincide with the original operator $\hat{\boldsymbol{\nu}} \Delta$ since $\hat{\boldsymbol{\nu}}$ does not commute with $M ; \hat{\boldsymbol{\nu}}$ is given by (3.12). Simple computation gives the resonant terms. Let $\nu_{1}$ and $\nu_{2}$ be the kinematic viscosity and the heat conductivity, respectively. We have in $V$-basis

$$
\exp \left(P^{d} M^{\prime} P^{d} N t\right) \hat{\boldsymbol{\nu}} \exp \left(-P^{d} M^{\prime} P^{d} N t\right)=\operatorname{diag}\left(\nu_{Q G}(n), \nu_{A G}(n), \nu_{A G}(n)\right)+\Re\left(2 N \omega_{n} t\right)
$$

where all the elements of the non-resonant matrix $\Re$ include factors $\exp \left( \pm i 2 N \omega_{n} t\right)$. Thus we obtain the diagonal resonant matrix $\tilde{\boldsymbol{\nu}}(n)=\operatorname{diag}\left(\nu_{Q G}(n), \nu_{A G}(n), \nu_{A G}(n)\right)$ in terms of the $\mathrm{QG}$ and AG viscosities $\nu_{Q G}$ and $\nu_{A G}$ given by (see BMN [11])

$$
\nu_{Q G}(n)=\nu_{2}+\left(\nu_{1}-\nu_{2}\right) \frac{\left|\check{n}^{\prime}\right|^{2}}{\left|\check{n}^{\prime}\right|^{2}+\eta^{2} \check{n}_{3}^{2}}, \nu_{A G}(n)=\nu_{1}+\left(\nu_{2}-\nu_{1}\right) \frac{\left|\check{n}^{\prime}\right|^{2}}{\left|\check{n}^{\prime}\right|^{2}+\eta^{2} \check{n}_{3}^{2}}
$$

where $\eta=f / N,\left|\check{n}^{\prime}\right|^{2}=n_{1}^{2}+n_{2}^{2} / a_{2}^{2}, \check{n}_{3}=n_{3} / a_{3}$. Clearly

$$
\begin{gathered}
\nu_{Q G}(n)=\nu_{1} \frac{\left|\check{n}^{\prime}\right|^{2}}{\left|\check{n}^{\prime}\right|^{2}+\eta^{2} \check{n}_{3}^{2}}+\nu_{2} \frac{\eta^{2} \check{n}_{3}^{2}}{\left|\check{n}^{\prime}\right|^{2}+\eta^{2} \check{n}_{3}^{2}}=\nu_{1} \frac{\left|\check{n}^{\prime}\right|^{2}}{\omega_{n}^{2}|\check{n}|^{2}}+\nu_{2} \frac{\eta^{2}\left|\check{n}_{3}\right|^{2}}{\omega_{n}^{2} \mid \check{n}^{2}}=\nu_{1} \frac{\phi_{n}^{2}}{\omega_{n}^{2}}+\nu_{2} \frac{\eta^{2} \xi_{n}^{2}}{\omega_{n}^{2}}, \\
\nu=\min \left(\nu_{1}, \nu_{2}\right) \leq \nu_{Q G}(n) \leq \max \left(\nu_{1}, \nu_{2}\right)
\end{gathered}
$$

and the same inequality holds for $\nu_{A G}(n)$.

The limit resonant equations are obtained by annihilating all terms in (3.20) which contain fast oscillating factors:

$$
\partial_{t} \mathbf{w}=\tilde{\mathbf{B}}(\mathbf{w}, \mathbf{w})-\tilde{\mathbf{A}} \mathbf{w}+\tilde{\mathbf{F}}
$$

where $\tilde{\mathbf{A}}=-\tilde{\boldsymbol{\nu}} \Delta$ and where $\tilde{\boldsymbol{\nu}}$ is the nonlocal linear matrix operator with symbol $\tilde{\boldsymbol{\nu}}(n)$ (when $\mathbf{F}$ depends on $N t$, the limit equations may include $\tilde{F}^{1}$ and $\tilde{F}^{2}$ resonant components). Clearly, when represented in Fourier modes, the operator $\tilde{\mathbf{B}}$ in the right-hand side of (3.25) has coefficients $\tilde{Q}_{k m n}^{i_{1} i_{2} i_{3}}$ in (3.20) and the limit resonant equations are

$$
\partial_{t} w_{n}^{i_{3}}=\sum_{n=k+m, i_{1}, i_{2}} \tilde{Q}_{k m n}^{i_{1} i_{2} i_{3}} w_{k}^{i_{1}} w_{m}^{i_{2}}-\tilde{A}_{n} w_{n}^{i_{3}}+\tilde{F}_{n}^{i_{3}}
$$

where $\tilde{A}_{n}=\tilde{\boldsymbol{\nu}}(n) \check{n}^{2}$ and the summation is over resonant terms. In equations (3.26) $\tilde{F}_{n}^{i_{3}}=\tilde{F}_{Q G, n}$ for $i_{3}=0$ and $\tilde{F}_{n}^{i_{3}}$ in the appropriate component of $\tilde{\mathbf{F}}_{A G, n}$ for $i_{3}=1,2$. Projecting (3.26) on the $Q G$ mode (with $i_{3}=0$ ) and projecting to the ageostrophic subspace we obtain separately the equations for QG and AG components. In this paper we usually consider the case $\mathbf{F}_{A G}$ non-resonant.

We note that projection of (3.25) on QG mode (which corresponds to zero eigenvalue of the linear problem) leads to the additional constraint $\omega_{n}^{\prime}=0$. Then the conditions $\pm \omega_{k}^{\prime} \pm \omega_{m}^{\prime} \pm \omega_{n}^{\prime}=0$ and $\omega_{n}^{\prime}=0$ reduce to 2-wave interactions $\omega_{k}=\omega_{m}$. For $\eta \neq 1$ the condition $\omega_{k}=\omega_{m}$ is equivalent to the condition $\left|\check{k}^{\prime}\right| /|\check{k}|=\left|\check{m}^{\prime}\right| /|\check{m}|$ (equivalently, $\phi_{k}=\phi_{m}$; see (3.4)). Clearly, the asymptotic limit equations (3.25) projected on QG mode involve only the coefficients $Q_{k m n}^{i_{1} i_{2} i_{3}}$ with $i_{3}=0(n=k+m)$. One trivial solution of $\omega_{k}^{\prime}=\omega_{m}^{\prime}$ is $\omega_{k}^{\prime}=\omega_{m}^{\prime}=0$ which corresponds to the $\mathrm{QG}$ coefficient $Q_{k m n}^{000}$. An important observation is that other terms involving the coefficients $Q_{k m n}^{i_{1} i_{2} 0}\left(i_{1} \neq 0\right.$ or $\left.i_{2} \neq 0\right)$ in equation (3.25) are annihilated for all $n, m$ when the resonance condition $\phi_{k}=\phi_{m}$ is used (see BMN [6,11]). Therefore the quasigeostrophic component of the resonant equation (3.25) completely decouples. This fact was proved in BMN $[6,11]$ by direct computation and also proved by Embid and Majda [26] using Ertel's theorem. 
The quasi-geostrophic equation (2.13) is given by

$$
\partial_{t} w_{n}^{0}=\mathbf{B}_{0}\left(w^{0}, w^{0}\right)_{n}-\tilde{A}_{n}^{Q G} w_{n}^{0}+\tilde{F}_{Q G, n}, \mathbf{B}_{0}\left(w^{0}, w^{0}\right)_{n}=-i \sum_{k+m=n} Q_{k m n}^{000} w_{k}^{0} w_{m}^{0}
$$

We introduce variables $\tilde{q}, \tilde{\mathbf{U}}_{Q G}, \tilde{\Psi}^{0}$ (quasi-geostrophic potential, velocity and stream function)

$$
\tilde{q}_{m}=\omega_{m}|\check{m}| w_{m}^{0}, \quad \tilde{\mathbf{U}}_{k}=\left[-k_{2} / a_{2}, k_{1}, 0,0\right] \tilde{\Psi}_{k}^{0}, \quad \tilde{\Psi}_{k}^{0}=\tilde{q}_{k} /\left(\omega_{k}^{2}|\check{k}|^{2}\right)
$$

Recalling that $\omega_{k}^{2}|\check{k}|^{2}=\left|\breve{k}^{\prime}\right|^{2}+\eta^{2} \breve{k}_{3}^{2}, \eta=f / N$, we have the familiar formula which relates $\tilde{\Psi}^{0}$ and $\tilde{q}$ in physical space

$$
-\left(\nabla_{h}^{2}+\eta^{2} \partial_{3}^{2}\right) \tilde{\Psi}^{0}=\tilde{q}
$$

Using (3.28), equation (3.27) can be written in the form:

$$
\partial_{t} \tilde{q}_{n}=-i \sum_{k+m=n}\left(\tilde{\mathbf{U}}_{k} \cdot m\right) \tilde{q}_{m}-\tilde{A}_{n}^{Q G} \tilde{q}_{n}+\omega_{n}|\check{n}| \tilde{F}_{n}^{0}
$$

$\tilde{q}(t, x)$ obeys in physical space the 3D quasi-geostrophic equations (see Bourgeois and Beale, [19], for inviscid case) where the viscous dissipation operator $\tilde{A}^{Q G}$ is linear pseudodifferential operator which in Fourier representation is multiplication by $\tilde{A}_{n}^{Q G}=\nu_{Q G}(n)|n|^{2}, \nu_{Q G}(n)$ given by $(3.22)$.

Thus, in the asymptotic limit equation $(3.25) \mathbf{w}=\left(w^{0}, w^{1}, w^{2}\right)$ splits into the limit quasi-geostrophic field $w^{0}(t)=\mathbf{w}_{Q G}$ satisfying (3.27) and found independently and into ageostrophic component $\mathbf{w}_{A G}=\left(w^{1}, w^{2}\right)$ which satisfies in general equations of the type:

$$
\partial_{t} \mathbf{w}_{A G}=\mathbf{B}_{2}\left(\mathbf{w}_{Q G}(t), \mathbf{w}_{A G}\right)+\mathbf{B}_{3}\left(\mathbf{w}_{A G}, \mathbf{w}_{A G}\right)-\tilde{A}^{A G} \mathbf{w}_{A G}+\tilde{\mathbf{F}}_{A G}
$$

And in Fourier representation:

$$
\partial_{t} \mathbf{w}_{A G, n}^{i_{3}}=\sum_{k+m=n, i_{1}, i_{2} ; \pm \omega_{k}^{\prime} \pm \omega_{m}^{\prime} \pm \omega_{n}^{\prime}=0} \tilde{Q}_{k m n}^{i_{1} i_{2} i_{3}} w_{k}^{i_{1}} w_{m}^{i_{2}}-\nu_{A G}(n)|\check{n}|^{2} w_{n}^{i_{3}}+\tilde{F}_{A G, n}^{i_{3}}
$$

where $i_{3} \neq 0 ; i_{1}, i_{2}=0,1,2$. These are the " $2 \frac{1}{2}$-dimensional" limit resonant equations in the Craya basis for the ageostrophic component $\mathbf{w}_{A G}=\left(w^{1}, w^{2}\right)$.

\section{Global Regularity of the limit Resonant equations}

In this section we prove global regularity of the limit resonant " $2 \frac{1}{2}$-dimensional" primitive Navier-Stokes equations for all domain aspect ratios and all 3 -waves resonances. The limit resonant operator $\tilde{\mathbf{B}}$ inherits properties of the operator B. This statement follows from the following

Lemma 4.1. Let $(\mathbf{u}, \mathbf{v}, \mathbf{w}) \in H_{3 / 4} \times H_{3 / 4} \times H_{1}$ given in Craya basis. Then

$$
(\tilde{\mathbf{B}}(\mathbf{u}, \mathbf{v}), \mathbf{w})=\lim _{N \rightarrow \infty} \frac{1}{2 \pi} \int_{0}^{2 \pi}(\mathbf{B}(N s, \mathbf{u}, \mathbf{v}), \mathbf{w}) \mathrm{d} s
$$

Here $\mathbf{u}, \mathbf{v}$ and $\mathbf{w}$ denote generic time-independent vectors in Craya basis. 
Proof. We introduce projections $\pi_{R}$ on finite-dimensional subspace of Fourier modes with $|n| \leq R$. We fix $(\mathbf{u}, \mathbf{v}, \mathbf{w}) \in H_{3 / 4} \times H_{3 / 4} \times H_{1}$. We put $\mathbf{u}_{R}=\pi_{R} \mathbf{u}$ and similarly for $\mathbf{v}$ and $\mathbf{w}$. Clearly,

$$
\begin{aligned}
(\mathbf{B}(N t, \mathbf{u}, \mathbf{v}), \mathbf{w})-(\tilde{\mathbf{B}}(\mathbf{u}, \mathbf{v}), \mathbf{w})= & {\left[(\mathbf{B}(N t, \mathbf{u}, \mathbf{v}), \mathbf{w})-\left(\mathbf{B}\left(N t, \mathbf{u}_{R}, \mathbf{v}_{R}\right), \mathbf{w}_{R}\right)\right] } \\
& +\left[\left(\mathbf{B}\left(N t, \mathbf{u}_{R}, \mathbf{v}_{R}\right), \mathbf{w}_{R}\right)-\left(\tilde{\mathbf{B}}\left(\mathbf{u}_{R}, \mathbf{v}_{R}\right), \mathbf{w}_{R}\right)\right] \\
& +\left[\left(\tilde{\mathbf{B}}\left(\mathbf{u}_{R}, \mathbf{v}_{R}\right), \mathbf{w}_{R}\right)-(\tilde{\mathbf{B}}(\mathbf{u}, \mathbf{v}), \mathbf{w}] .\right.
\end{aligned}
$$

The operators $\mathbf{B}$ and $\tilde{\mathbf{B}}$ are continuous on $H_{3 / 4} \times H_{3 / 4} \times H_{1}$. Moreover, since the unitary Poincaré propagator $\mathbf{E}(N t)$ preserves all Sobolev norms, the operator $\mathbf{B}(N t, \mathbf{u}, \mathbf{v})$ is continuous uniformly in $N t$. Therefore, the first and third brackets in the right-hand side tend to zero as $R \rightarrow \infty$. Let $\epsilon>0$, we find such $R$ that the absolute values of the first and third brackets are less than $\epsilon$.

After that we consider the second bracket

$$
\begin{gathered}
{\left[\left(\mathbf{B}\left(N t, \mathbf{u}_{R}, \mathbf{v}_{R}\right), \mathbf{w}_{R}\right)-\left(\tilde{\mathbf{B}}\left(\mathbf{u}_{R}, \mathbf{v}_{R}\right), \mathbf{w}_{R}\right)\right]=} \\
\left(\mathbf{B}\left(N t, \mathbf{u}_{R}, \mathbf{v}_{R}\right)-\tilde{\mathbf{B}}\left(\mathbf{u}_{R}, \mathbf{v}_{R}\right), \mathbf{w}_{R}\right)=\left(\mathbf{B}_{o s c}\left(N t, \mathbf{u}_{R}, \mathbf{v}_{R}\right), \mathbf{w}_{R}\right) .
\end{gathered}
$$

Since $\mathbf{B}_{\text {osc }}$ contains only non-resonant terms, we obtain integrating by parts like in $[8,14]$ that

$$
\frac{1}{2 \pi} \int_{0}^{2 \pi}\left(\mathbf{B}_{o s c}\left(N s, \mathbf{u}_{R}, \mathbf{v}_{R}\right), \mathbf{w}_{R}\right) \mathrm{d} s=O(1 / N) \rightarrow 0 \text { as } N \rightarrow \infty
$$

Therefore, the integrals of all three brackets are less than $\epsilon$ when $N$ is large, and the lemma is proven.

Corollary 4.1. Let $\sigma \geq 1$ and $\mathbf{w}$ be the Craya vector variable, $\mathbf{w}=\left(w^{0}, w^{1}, w^{2}\right)$ ( $w^{0}$ corresponds to QG modes and $w^{1}, w^{2}$ correspond to AG modes). Then

$$
\left(\tilde{\mathbf{B}}\left(\mathbf{w},(-\Delta)^{\sigma / 2} \mathbf{w}\right),(-\Delta)^{\sigma / 2} \mathbf{w}\right)=0 .
$$

Proof. From Lemma 4.1, it suffices to prove the similar identity for the general operator $\mathbf{B}(N t, \mathbf{v}, \mathbf{v})$ in equations (3.18)-(3.20) for the non-averaged equations (3.20) written in Fourier space in the Craya basis. Using equation (3.13)

$$
\begin{array}{r}
\left(\mathbf{B}\left(N t, \mathbf{v},(-\Delta)^{\sigma / 2} \mathbf{v}\right),(-\Delta)^{\sigma / 2} \mathbf{v}\right)=\sum_{k+m+n=0 ; i_{1}, i_{2}, i_{3} ; l=1, \ldots, 8} c_{l} \exp \left(i D_{l}(k, m, n) N t\right) Q_{k m n}^{i_{1} i_{2} i_{3}} v_{k}^{i_{1}} v_{m}^{i_{2}}|\check{m}|^{\sigma}|\check{n}|^{\sigma} v_{n}^{i_{3}} \\
=\sum_{k+m+n=0 ; i_{1}, i_{2}, i_{3} ; l=1, \ldots, 8} c_{l} \exp \left(i D_{l}(k, m, n) N t\right)\left(q_{i_{1} k} \cdot \check{m}\right)\left(q_{i_{2} m} \cdot q_{i_{3} n}\right)|\check{m}|^{\sigma}|\check{n}|^{\sigma} v_{k}^{i_{1}} v_{m}^{i_{2}} v_{n}^{i_{3}} \\
=\sum_{k+m+n=0 ; i_{1}, i_{2}, i_{3} ; l=1, \ldots, 8} c_{l} \exp \left(i D_{l}(k, m, n) N t\right)\left(q_{i_{1} k} \cdot \check{n}\right)\left(q_{i_{3} n} \cdot q_{i_{2} m}\right)|\check{m}|^{\sigma}|\check{n}|^{\sigma} v_{k}^{i_{1}} v_{m}^{i_{2}} v_{n}^{i_{3}} \\
=-\sum_{k+m+n=0 ; i_{1}, i_{2}, i_{3} ; l=1, \ldots 8} c_{l} \exp \left(i D_{l}(k, m, n) N t\right)\left(q_{i_{1} k} \cdot(\check{k}+\check{m})\right)\left(q_{i_{3} n} \cdot q_{i_{2} m}\right)|\check{m}|^{\sigma}|\check{n}|^{\sigma} v_{k}^{i_{1}} v_{m}^{i_{2}} v_{n}^{i_{3}} \\
=-\sum_{k+m+n=0 ; i_{1}, i_{2}, i_{3} ; l=1, \ldots, 8} c_{l} \exp \left(i D_{l}(k, m, n) N t\right)\left(q_{i_{1} k} \cdot \check{m}\right)\left(q_{i_{3} n} \cdot q_{i_{2} m}\right)|\check{m}|^{\sigma}|\check{n}|^{\sigma} v_{k}^{i_{1}} v_{m}^{i_{2}} v_{n}^{i_{3}} \\
=-\left(\mathbf{B}\left(N t, \mathbf{v},(-\Delta)^{\sigma / 2} \mathbf{v}\right),(-\Delta)^{\sigma / 2} \mathbf{v}\right)
\end{array}
$$

where in the above sum we interchanged indices $m$ and $n, i_{2}$ and $i_{3}$ and used the divergence free condition $\breve{k} \cdot q_{i_{1} k}=0$. Here $c_{l}$ are absolute constants indexed by $i_{1}, i_{2}, i_{3}$ with values $\pm 1 / 8$ (cf. (3.17), (3.19)). We use 
$(\mathbf{E}(N t))^{*}=\mathbf{E}(-N t)$ to ensure symmetry of the terms $(\mathbf{E}(-N t) \mathbf{v})_{m}$ and $(\mathbf{E}(-N t) \mathbf{v})_{n}$. Then from (4.3) we have

$$
\left(\mathbf{B}\left(N t, \mathbf{v},(-\Delta)^{\sigma / 2} \mathbf{v}\right),(-\Delta)^{\sigma / 2} \mathbf{v}\right)=0 .
$$

Equation (4.2) follows from (4.4) and Lemma 4.1.

We follow with the estimate for the resonant operator $\tilde{\mathbf{B}}(\mathbf{w}, \mathbf{w})$ in Craya basis:

Corollary 4.2. Let $\sigma \geq 1$ and $\mathbf{w}$ be the Craya vector variable. Then

$$
|(\tilde{\mathbf{B}}(\mathbf{w}, \mathbf{w}),(-\Delta) \mathbf{w})| \leq C_{\tilde{B}} \sum_{k+m+n=0, \pm \omega_{k}^{\prime} \pm \omega_{m}^{\prime} \pm \omega_{n}^{\prime}=0}|\check{k}|\left|\mathbf{w}_{k}\right||\check{m}|\left|\mathbf{w}_{m}\right||\check{n}|\left|\mathbf{w}_{n}\right| .
$$

Proof. Recall that the Craya basis vectors $q^{\prime} s$ are normalized with norm one. We first prove such an estimate for the non-resonant general operator $\mathbf{B}(N t, \mathbf{v}, \mathbf{v})$ for every fixed $t$ (but without the restriction $\pm \omega_{k}^{\prime} \pm \omega_{m}^{\prime} \pm \omega_{n}^{\prime}=0$ ). We have using (4.4) with $\sigma=1$

$$
\begin{array}{r}
(\mathbf{B}(N t, \mathbf{v}, \mathbf{v}),(-\Delta) \mathbf{v})=\sum_{k+m+n=0 ; i_{1}, i_{2}, i_{3} ; l=1, \ldots, 8} c_{l} \exp \left(i D_{l}(k, m, n) N t\right)\left(q_{i_{1} k} \cdot \check{m}\right)\left(q_{i_{2} m} \cdot q_{i_{3} n}\right)|\check{n}|^{2} v_{k}^{i_{1}} v_{m}^{i_{2}} v_{n}^{i_{3}} \\
=\sum_{k+m+n=0 ; i_{1}, i_{2}, i_{3} ; l=1, \ldots, 8} c_{l} \exp \left(i D_{l}(k, m, n) N t\right)\left(q_{i_{1} k} \cdot \check{m}\right)\left(q_{i_{2} m} \cdot q_{i_{3} n}\right)|\check{n}|(|\check{n}|-|\check{m}|) v_{k}^{i_{1}} v_{m}^{i_{2}} v_{n}^{i_{3}} .
\end{array}
$$

Note that ||$\check{n}|-| \check{m}||=|| \check{k}+\check{m}|-| \check{m}|\leq 7| \check{k} \mid$, to get:

$$
|(\mathbf{B}(N t, \mathbf{v}, \mathbf{v}),(-\Delta) \mathbf{v})| \leq C_{B} \sum_{k+m+n=0}|\check{k}|\left|\mathbf{v}_{k}\right||\check{m}|\left|\mathbf{v}_{m}\right|\left|\check{n} \| \mathbf{v}_{n}\right| .
$$

The same estimate follows for $\tilde{\mathbf{B}}(\mathbf{w}, \mathbf{w})$, from the skew-symmetry Corollary 4.1 and from averaging equation (4.6); this only further restricts $k, m, n$ interactions to the set $\pm \omega_{k}^{\prime} \pm \omega_{m}^{\prime} \pm \omega_{n}^{\prime}=0$ where $\omega_{n}^{\prime}=0$ for quasi-geostrophic modes and $\omega_{n}^{\prime}=\omega_{n}$ for ageostrophic modes, and similarly for $k, m$. In equation (3.20), the resonant operators $\tilde{Q}_{k m n}^{i_{1} i_{2} i_{3}}$ are first order Fourier integral operators. Then for $\tilde{\mathbf{B}}(\mathbf{w}, \mathbf{w})$ we obtain:

$$
|(\tilde{\mathbf{B}}(\mathbf{w}, \mathbf{w}),(-\Delta) \mathbf{w})| \leq C_{\tilde{B}} \sum_{k+m+n=0, \pm \omega_{k}^{\prime} \pm \omega_{m}^{\prime} \pm \omega_{n}^{\prime}=0}|\check{k}|\left|\mathbf{w}_{k}\right||\check{m}|\left|\mathbf{w}_{m}\right||\check{n}|\left|\mathbf{w}_{n}\right| .
$$

Remark 4.1. Since $\mathbf{w}_{A G}$ is orthogonal to $\mathbf{w}_{Q G}$ (orthogonality of the q's) and from the skew-symmetry of $\mathbf{B}_{2}$ proven in [12] we also have:

$$
\left(\mathbf{B}_{3}\left(\mathbf{w}_{A G},(-\Delta)^{\sigma / 2} \mathbf{w}_{A G}\right),(-\Delta)^{\sigma / 2} \mathbf{w}_{A G}\right)=0
$$

and the estimate (4.8) is valid for $\left|\left(\mathbf{B}_{2}\left(\mathbf{w}_{Q G}, \mathbf{w}_{A G}\right)+\mathbf{B}_{3}\left(\mathbf{w}_{A G}, \mathbf{w}_{A G}\right),(-\Delta) \mathbf{w}_{A G}\right)\right|$.

Remark 4.2. The above estimate (4.8) will be used together with the Lemma 4.2 on restricted convolutions to obtain the global regularity in $H_{1}$ for equations (3.25-3.26).

We recall some facts on the geometry of resonances (see [12]). We denote

$$
\eta=f / N, \theta_{1}=1 / a_{1}^{2}, \theta_{2}=1 / a_{2}^{2}, \theta_{3}=1 / a_{3}^{2} .
$$

Let $K$ denote the set of resonant wavenumbers $k, m, n$ for given $\eta, a_{1}, a_{2}, a_{3}$ :

$$
K=\left\{ \pm \omega_{k}^{\prime} \pm \omega_{m}^{\prime} \pm \omega_{n}^{\prime}=0\right\}
$$


where $\omega_{n}^{\prime}=0$ for QG modes and $\omega_{n}^{\prime}=\omega_{n}$ for AG modes and similarly for $k$ and $m$. We also define the set of strict 3 -waves resonances

$$
K^{*}=\left\{ \pm \omega_{k} \pm \omega_{m} \pm \omega_{n}=0, \omega_{k} \omega_{m} \omega_{n} \neq 0\right\} .
$$

For non-generic values of these parameters, the limit inviscid equations for the ageostrophic field $\mathbf{w}_{A G}$ are nonlinear:

$$
\begin{aligned}
& \partial_{t} \mathbf{w}_{A G}=\mathbf{B}_{2}\left(\mathbf{w}_{Q G}(t), \mathbf{w}_{A G}\right)+\mathbf{B}_{3}\left(\mathbf{w}_{A G}, \mathbf{w}_{A G}\right) \\
& \mathbf{B}_{3}\left(\mathbf{w}_{A G}, \mathbf{w}_{A G}\right)^{i_{3}}=\sum_{\substack{ \pm \omega_{k} \pm \omega_{m} \pm \omega_{n}=0 \\
k+m+n=0 \\
i_{1}, i_{2}=1 \text { or } 2}} \tilde{Q}_{k m n}^{i_{1} i_{2} i_{3}} w_{k}^{i_{1}} w_{m}^{i_{2}} ;
\end{aligned}
$$

the coefficients $\tilde{Q}_{k m n}^{i_{1} i_{2} i_{3}}$ can easily be computed in the Craya cyclic basis and are not detailed in this section for conciseness sake. For $\mathbf{B}_{3}$, the domain of summation is determined by the set $K^{*}$ of strict 3 -wave resonances. This set is defined by condition $\pm \omega_{n} \pm \omega_{m} \pm \omega_{k}=0$ where $\omega_{n}, \omega_{m}, \omega_{k} \neq 0$ depends on $\left(\eta, 1 / a_{2}^{2}, 1 / a_{3}^{2}\right)=\left(\eta, \theta_{2}, \theta_{3}\right)$; that is $K^{*}=K^{*}\left(\eta, \theta_{2}, \theta_{3}\right)\left(\theta_{1}=1\right.$ without loss of generality). For every fixed $\theta_{2}=1 / a_{2}^{2}$ and $\theta_{3}=1 / a_{3}^{2}$, the set $K^{*}\left(\eta, \theta_{2}, \theta_{3}\right)$ is not empty when $\eta \in \Theta^{*}\left(\theta_{2}, \theta_{3}\right)$; the singular set $\Theta^{*}\left(\theta_{2}, \theta_{3}\right)$ is countable. We call it a strict 3 -wave resonant set. When $\eta \in \Theta^{*}\left(\theta_{2}, \theta_{3}\right)$ is strictly resonant, $\mathbf{B}_{3}$ is non-zero and depends strongly on $\eta$; the sets $K^{*}\left(\eta, \theta_{2}, \theta_{3}\right)$ with different, but close $\eta$ do not intersect (a nontrivial result from the study of the small divisor problem, cf. [12]). This implies that the operator $\mathbf{B}_{3}$ depends on resonant $\eta$ discontinuously, at every point $\eta \in \Theta^{*}\left(\theta_{2}, \theta_{3}\right)$ is a point of discontinuity of the operator $\mathbf{B}_{3}$. Since $\mathbf{B}_{3}$ is not zero, solutions of the limit system with general initial data discontinuously depend on $\eta$ as well. As solutions of the original 3D primitive equations depend on $\eta$ continuously (on a small time interval $\left[0, T_{1}\right]$ ), the convergence to solutions of the limit equations cannot be uniform in $\eta, a_{2}, a_{3}$. When $(k, m, n) \notin K^{*}\left(i . e . \eta \notin \Theta^{*}\right)$, only the catalytic operator $\mathbf{B}_{2}$ is present. We refer to $[10,12]$ and [15] for an extensive study of the analytic form and properties of $\mathbf{B}_{2}$.

We present new estimates for the nonlinear " $2 \frac{1}{2}$-dimensional" operator $\mathbf{B}_{3}$ corresponding to strict 3 -waves resonances which do ensure global existence of strong solutions of the limit ageostrophic viscous equations (3.313.32 ) and, consequently, equations (3.25-3.26) for all domain parameters and all 3 -waves resonances. The following theorem which will be proven below provides the main estimate for the resonant operator $\mathbf{B}_{3}$ for the 'worst' case of all interactions on the " $2 \frac{1}{2}$-dimensional" interaction manifold $K^{*}$ :

Theorem 4.1. Let $\mathbf{w}_{A G}\left(x_{1}, x_{2}, x_{3}\right) \in H_{2}$ (Sobolev space of periodic vector fields with zero mean). Then the following estimate holds

$$
\left|\left(\mathbf{B}_{3}\left(\mathbf{w}_{A G}, \mathbf{w}_{A G}\right),(-\Delta) \mathbf{w}_{A G}\right)\right| \leq C_{I I I}|| \mathbf{w}_{A G}\left\|_{2}\right\| \mathbf{w}_{A G} \|_{1}^{2} .
$$

Remark 4.3. Estimate (4.14) is of the same type as the classical estimate of Ladyzhenskaya [36] in the twodimensional case with Dirichlet boundary conditions. For the periodic boundary conditions in 2D it is wellknown that the analog to the left hand side of (4.14) is identically zero ([23]). Of course, in (4.14) the divergence free vector field $\mathbf{w}_{A G}\left(x_{1}, x_{2}, x_{3}\right)$ and the Sobolev spaces $H_{\alpha}$ are three-dimensional with space variables $x_{1}, x_{2}$ and $x_{3}$.

From the estimate (4.14) we immediately obtain in a standard way ( $c f .[16,23,48,49])$ (note that if the force $\mathbf{F}(t, x)$ in the original equation does not depend on $N$ and $f=2 \Omega$, then $\left.\tilde{\mathbf{F}}_{A G}=0\right)$ :

Theorem 4.2. Let $\nu_{1}, \nu_{2}>0, \nu=\min \left(\nu_{1}, \nu_{2}\right),\left\|\mathbf{w}_{A G}(0)\right\|_{\alpha} \leq M_{\alpha}, 1 \geq \alpha>3 / 4 ; \tilde{\mathbf{F}}_{A G}$ satisfies (4.15) with $\alpha=1$ :

$$
\sup _{T} \int_{T}^{T+1}\left\|\tilde{\mathbf{F}}_{A G}\right\|_{\alpha-1}^{2} \mathrm{~d} t \leq M_{\alpha F}^{2}
$$


Then there exists a unique regular solution $\mathbf{w}_{A G}(t)$ of " $2 \frac{1}{2}$-dimensional" primitive Navier-Stokes (3.31-3.32), $\left\|\mathbf{w}_{A G}(t)\right\|_{1} \leq M_{1}^{\prime}\left(\nu, M_{1 F}, M_{\alpha}, a_{1}, a_{2}, a_{3}\right)$ for all $t \geq 0$.

Proof. A local regularity solution to the " $2 \frac{1}{2}$-dimensional" equations (3.31-3.32) exists on a small interval of time $0 \leq t \leq t_{1}$ (see BMN [8]) and belongs to $H_{1}$ for $0<t<t_{1}$ thanks to the smoothing property which follows from

$$
\frac{\nu_{A G}}{2} \int_{0}^{t}\left\|\mathbf{w}_{A G}\right\|_{1+\gamma}^{2} d \tau+\left\|\mathbf{w}_{A G}(t)\right\|_{\gamma}^{2} \leq C(t), 0 \leq t<t_{1} .
$$

Therefore, it is sufficient to consider $\gamma=1$.

Multiplying equation (3.31) for $\mathbf{w}_{A G}$ by $(-\Delta) \mathbf{w}_{A G}$ we obtain

$$
\begin{aligned}
\partial_{t}\left\|\mathbf{w}_{A G}\right\|_{1}^{2}= & -2 \nu_{A G}\left\|\mathbf{w}_{A G}\right\|_{2}^{2}+2\left(\mathbf{B}_{3}\left(\mathbf{w}_{A G}, \mathbf{w}_{A G}\right),(-\Delta) \mathbf{w}_{A G}\right) \\
& +2\left(\mathbf{B}_{2}\left(\mathbf{w}_{Q G}, \mathbf{w}_{A G}\right),(-\Delta) \mathbf{w}_{A G}\right)+2\left(\tilde{\mathbf{F}}^{A G},(-\Delta) \mathbf{w}_{A G}\right) .
\end{aligned}
$$

For the 3-wave resonant operator $\mathbf{B}_{3}\left(\mathbf{w}_{A G}, \mathbf{w}_{A G}\right)$ we have from Theorem 4.1

$$
\left|\left(\mathbf{B}_{3}\left(\mathbf{w}_{A G}, \mathbf{w}_{A G}\right),(-\Delta) \mathbf{w}_{A G}\right)\right| \leq C_{\mathrm{III}}|| \mathbf{w}_{A G}\left\|_{2}|| \mathbf{w}_{A G}\right\|_{1}^{2} .
$$

We have according to [12]

$$
\left|\left(\mathbf{B}_{2}\left(\mathbf{w}_{Q G}, \mathbf{w}_{A G}\right),(-\Delta) \mathbf{w}_{A G}\right)\right| \leq C_{\mathrm{II}}|| \tilde{\mathbf{U}}_{Q G}\left\|_{1}\right\| \mathbf{w}_{A G} \|_{1}^{2}
$$

where the $Q G$ velocity $\tilde{\mathbf{U}}_{Q G}$ was defined in equation (3.28). Estimates for the viscous QG equation are derived in [12]. Using the above estimates, a standard Gronwall inequality yields the estimate in $H_{1}$ for all $t$, and uniqueness of the solutions $\mathbf{w}_{A G}$ follows in a standard way $(c f .[23,49])$. Theorem 4.2 is proven.

Remark 4.4. Using Theorems 4.1 and 4.2 one can develop regularity theory for solutions of " $2 \frac{1}{2}$-dimensional" Navier-Stokes equations in $H_{\gamma}, \gamma>1$ spaces. This is done similar to the well-known higher regularity theory for sufficiently regular solutions of three-dimensional Navier-Stokes equations (see Temam [49]).

Now we prove Theorem 4.1; it is based on the following Lemma on Restricted Convolutions. Here without loss of generality we put $\theta_{1}=\theta_{2}=\theta_{3}=1$.

Lemma 4.2 (Lemma on Restricted Convolutions). Let $\chi(k, m, n)$ be the characteristic function of some set $K^{*}$ in $\left(\mathbf{Z}^{3}\right)^{3}$ such that $\chi(k, m, n)=\chi(m, k, n)=\chi(k, n, m)$ is symmetric. Let $\alpha \geq 0, \beta$ fixed and

$$
\sup _{n} \sum_{k: k+m+n=0, k \in \Sigma_{i}} \chi(k, m, n)|k|^{-\alpha} \leq C_{0} 2^{i \beta}
$$

for every $i=0,1,2, \ldots$ where

$$
\Sigma_{i}=\left\{k=\left(k_{1}, k_{2}, k_{3}\right) \quad\left|\quad 2^{i} \leq\right| k\left|<2^{i+1},\right| k \mid=\sqrt{k_{1}^{2}+k_{2}^{2}+k_{3}^{2}}\right\} .
$$

Then for any sequence $u_{n}$ with $u_{(0,0,0)}=0$ :

$$
\sum_{k+m+n=0}\left|u_{k}\right|\left|u_{m}\right|\left|u_{n}\right| \chi(k, m, n) \leq C\left(\sum_{n}|n|^{\beta}\left|u_{n}\right|^{2}\right)^{1 / 2}\left(\sum_{k}|k|^{\alpha}\left|u_{k}\right|^{2}\right)^{1 / 2}\left(\sum_{m}\left|u_{m}\right|^{2}\right)^{1 / 2}
$$

where $C=6 \sqrt{2 C_{0}}$. 
Proof. Let $\alpha \geq 0$. We first give the proof for $\beta \geq 0$. Since the left sum in equation (4.22) is symmetric with respect to $k, m, n$

$$
\sum_{k+m+n=0}\left|u_{k} u_{m} u_{n}\right| \chi(k, m, n) \leq 6 \sum_{k+m+n=0,|n| \geq|k| \geq|m|}\left|u_{k} u_{m} u_{n}\right| \chi(k, m, n)
$$

and it is sufficient to take $k, m, n$ such that $|n| \geq|k| \geq|m|$. After that, we apply the technique of dyadic decomposition of Littlewood-Paley (Stein [46]). We estimate

$$
S=\sum_{k+m+n=0,|n| \geq|k| \geq|m|}\left|u_{k} u_{m} u_{n}\right| \chi(k, m, n)=\sum_{i} \sum_{n}\left|u_{n}\right| \sum_{k \in \Sigma_{i},|n| \geq|k| \geq|m|}\left|u_{k} u_{-k-n}\right| \chi(k,-k-n, n) .
$$

Since $|n| \geq|k| \geq|m|$ and $k+m+n=0$, we have $2|k| \geq|n| \geq|k|$. Therefore,

$$
\begin{aligned}
S & \leq \sum_{i} \sum_{n \in \Sigma_{i} \cup \Sigma_{i+1}}\left|u_{n}\right| \sum_{k \in \Sigma_{i}}\left|u_{k} u_{-k-n}\right| \chi(k,-k-n, n) \\
& \leq \sum_{i} \sum_{n \in \Sigma_{i} \cup \Sigma_{i+1}}\left|u_{n}\right|\left(\sum_{k \in \Sigma_{i}}\left|u_{k} u_{-k-n}\right|^{2}|k|^{\alpha}\right)^{1 / 2}\left(\sum_{k \in \Sigma_{i}}|k|^{-\alpha} \chi(k,-k-n, n)\right)^{1 / 2} \\
& \leq \sum_{i}\left(\sum_{n \in \Sigma_{i} \cup \Sigma_{i+1}}\left|u_{n}\right|^{2}\right)^{1 / 2}\left(\sum_{n \in \Sigma_{i} \cup \Sigma_{i+1}} \sum_{k \in \Sigma_{i}}\left|u_{k} u_{-k-n}\right|^{2}|k|^{\alpha}\right)^{1 / 2} \\
& \sup _{n}\left(\sum_{k \in \Sigma_{i}}|k|^{-\alpha} \chi(k,-k-n, n)\right)^{1 / 2} \\
& \leq C_{0}^{1 / 2} \sum_{i}\left(\sum_{n \in \Sigma_{i} \cup \Sigma_{i+1}}\left|u_{n}\right|^{2}\right)^{1 / 2}\left(\sum_{n \in \Sigma_{i} \cup \Sigma_{i+1}} \sum_{k \in \Sigma_{i}}\left|u_{k} u_{-k-n}\right|^{2}|k|^{\alpha}\right)^{1 / 2} 2^{\beta i / 2} \\
& \leq C_{0}^{1 / 2} \sum_{i}\left(\sum_{n \in \Sigma_{i} \cup \Sigma_{i+1}}\left|u_{n}\right|^{2} 2^{\beta i}\right)^{1 / 2}\left(\sum_{m} \sum_{k \in \Sigma_{i}}\left|u_{k} u_{m}\right|^{2}|k|^{\alpha}\right)^{1 / 2} \\
& \leq C_{0}^{1 / 2} \sum_{i}\left(\sum_{n \in \Sigma_{i} \cup \Sigma_{i+1}}\left|u_{n}\right|^{2} 2^{\beta i}\right)^{1 / 2}\left(\sum_{k \in \Sigma_{i}}\left|u_{k}\right|^{2}|k|^{\alpha}\right)^{1 / 2}\left(\sum_{m}\left|u_{m}\right|^{2}\right)^{1 / 2} .
\end{aligned}
$$

Therefore,

$$
\begin{aligned}
S & \leq C_{0}^{1 / 2} \sum_{i}\left(\sum_{n \in \Sigma_{i} \cup \Sigma_{i+1}}|n|^{\beta}\left|u_{n}\right|^{2}\right)^{1 / 2}\left(\sum_{k \in \Sigma_{i}}\left|u_{k}\right|^{2}|k|^{\alpha}\right)^{1 / 2}\left(\sum_{m}\left|u_{m}\right|^{2}\right)^{1 / 2} \\
& \leq C_{0}^{1 / 2}\left(\sum_{i} \sum_{n \in \Sigma_{i} \cup \Sigma_{i+1}}|n|^{\beta}\left|u_{n}\right|^{2}\right)^{1 / 2}\left(\sum_{i} \sum_{k \in \Sigma_{i}}\left|u_{k}\right|^{2}|k|^{\alpha}\right)^{1 / 2}\left(\sum_{m}\left|u_{m}\right|^{2}\right)^{1 / 2} \\
& \leq\left(2 C_{0}\right)^{1 / 2}\left(\sum_{n}|n|^{\beta}\left|u_{n}\right|^{2}\right)^{1 / 2}\left(\sum_{k}|k|^{\alpha}\left|u_{k}\right|^{2}\right)^{1 / 2}\left(\sum_{m}\left|u_{m}\right|^{2}\right)^{1 / 2} .
\end{aligned}
$$


Considering similarly other permutations of $|k|,|m|$ and $|n|$ we obtain $(4.22)$ with $C=6 \sqrt{2 C_{0}}$. The proof extends to $\beta<0$ with a different constant $C$.

We note that one obtains similar results for general $\theta_{1}, \theta_{2}, \theta_{3}$ bounded away from 0 and $+\infty$. Then the constants depend on $\theta_{1}, \theta_{2}, \theta_{3}$.

Proof of Theorem 4.1. From Corollary 4.2 we obtain the following inequality

$$
\left|\left(\mathbf{B}_{3}\left(\mathbf{w}_{A G}, \mathbf{w}_{A G}\right),(-\Delta) \mathbf{w}_{A G}\right)\right| \leq c^{\prime} \sum_{k+m+n=0}|\check{k}|\left|\mathbf{w}_{A G, k}\right||\check{m}|\left|\mathbf{w}_{A G, m}\right||\check{n}|\left|\mathbf{w}_{A G, n}\right| \chi(k, m, n) .
$$

Here $\chi(k, m,-n)$ is the characteristic function of the resonant set $K^{*}$ of strict three-wave resonances defined in (4.12). This set lies in the manifold of solutions of a polynomial equation $P(k, m, n)=0$. Indeed, we have the identity (similarly, for other combinations of + and - signs):

$$
1 /\left(\omega_{k}+\omega_{n}-\omega_{m}\right)=\frac{\left(\omega_{k}+\omega_{m}+\omega_{n}\right)\left(-\omega_{k}+\omega_{m}+\omega_{n}\right)\left(-\omega_{n}+\omega_{m}+\omega_{k}\right)}{\left(\omega_{k}+\omega_{m}+\omega_{n}\right)\left(\omega_{k}-\omega_{m}+\omega_{n}\right)\left(-\omega_{k}+\omega_{m}+\omega_{n}\right)\left(\omega_{k}+\omega_{m}-\omega_{n}\right)} .
$$

The denominator is $-P=\left(\left(\omega_{k}\right)^{2}+\left(\omega_{m}\right)^{2}-\left(\omega_{n}\right)^{2}\right)^{2}-4\left(\omega_{k}\right)^{2}\left(\omega_{m}\right)^{2} . P$ is a polynomial of degree 2 of $\lambda=\eta^{2}$. We write $\omega_{k}$ in the form (see Eq. (3.4)):

$$
\omega_{k}=\sqrt{\chi_{k}+\lambda\left(1-\chi_{k}\right)}, \chi_{k}=\left|\check{k}^{\prime}\right|^{2} /|\check{k}|^{2}=\phi_{k}^{2}, 1-\chi_{k}=\xi_{k}^{2} .
$$

The polynomial takes the form (with $\left.\lambda=\eta^{2}\right) P=P_{2} \lambda^{2}+P_{1} \lambda+P_{0}$, where

$$
\begin{aligned}
& P_{2}=\chi_{k}^{2}+\chi_{m}^{2}+\chi_{n}^{2}-2 \chi_{k} \chi_{n}-2 \chi_{k} \chi_{m}-2 \chi_{m} \chi_{n}-3+2\left(\chi_{k}+\chi_{m}+\chi_{n}\right) ; \\
& P_{1}=-2\left(\chi_{k}^{2}+\chi_{m}^{2}+\chi_{n}^{2}-2 \chi_{k} \chi_{n}-2 \chi_{k} \chi_{m}-2 \chi_{m} \chi_{n}+\chi_{k}+\chi_{m}+\chi_{n}\right) \\
& P_{0}=\chi_{k}^{2}+\chi_{m}^{2}+\chi_{n}^{2}-2 \chi_{k} \chi_{n}-2 \chi_{k} \chi_{m}-2 \chi_{m} \chi_{n} .
\end{aligned}
$$

Instead of considering $P$ as a polynomial in $\eta^{2}$, we renormalize it as

$$
\Pi(k, m, n)=|\check{k}|^{4}|\check{m}|^{4}|\check{n}|^{4} P(k, m, n) .
$$

where $\Pi$ is a homogeneous polynomial of degree 12 in the variables $k, m, n$ and $\eta$ is considered as a parameter. For given $\eta, \theta_{1}, \theta_{2}, \theta_{3}, \Pi(k, m, n)=0$ is equivalent to $(k, m, n) \in K^{*}$ (vice-versa, fixing $k, m, n$ as parameters, and solving for $\eta$ as a function of $\theta_{1}=1, \theta_{2}, \theta_{3}$ defines the singular values of $\left.\eta \in \Theta^{*}\left(\theta_{2}, \theta_{3}\right)\right)$. It follows that for fixed $\eta, \theta_{2}, \theta_{3}, \Pi(k,-k-n,-n)$ is a polynomial of degree at most eight in $k_{3}$. The leading power in $k_{3}^{8}$ is:

$$
-k_{3}^{8}\left(\left|n^{\prime}\right|^{2}+\eta^{2} n_{3}^{2}\right)\left(3 \eta^{2} n_{3}^{2}+\left(4 \eta^{2}-1\right)\left|n^{\prime}\right|^{2}\right)
$$

where $m$ was eliminated via $m=-k-n$. If this leading term (4.26) is not zero, there are at most eight $k_{3}$ satisfying $\Pi(k,-m-n,-n)=0$ for given $k_{1}, k_{2}, n$; this holds provided that

$$
3 \eta^{2} n_{3}^{2}+\left(4 \eta^{2}-1\right)\left|n^{\prime}\right|^{2} \neq 0
$$

Note that if $n^{\prime}=0$, the condition (4.27) is trivially satisfied as $n \neq 0$. Also, the condition is satisfied whenever $4 \eta^{2}-1 \geq 0$. If the condition (4.27) is not satisfied, i.e. if $n$ belongs to the manifold:

$$
3 \eta^{2} n_{3}^{2}+\left(4 \eta^{2}-1\right)\left|n^{\prime}\right|^{2}=0, n^{\prime} \neq 0,
$$

then we must verify that the polynomial $\Pi$ is not identically null. This is not trivial, as one verifies that the coefficient of $k_{3}^{7}$ is null under the condition (4.28). Under the latter condition the coefficient of $k_{3}^{6}$ reduces to:

$$
k_{3}^{6} \frac{\left|n^{\prime}\right|^{4}}{\eta^{2}}\left(-4 \eta^{4} \frac{1-4 \eta^{2}}{\eta^{2}}\left|n^{\prime}\right|^{2}+\frac{8}{9} \frac{\left(\eta^{2}-1\right)^{3}}{\eta^{2}}\left(\left|m^{\prime}\right|^{2}+\left|k^{\prime}\right|^{2}\right)\right)
$$


which is strictly negative whenever $0<\eta^{2} \leq 1 / 4$ and $n^{\prime} \neq 0$. Therefore, the polynomial $\Pi\left(k_{3}\right)$ does not vanish for any value of admissible parameters, for fixed $k_{1}, k_{2}, n$. Then there are at most eight $k_{3}$ satisfying $\chi(k,-k-n,-n)=0$.

Now we estimate the sum in (4.20) with $\alpha=1$ as follows

$$
\sum_{2^{i} \leq|k|<2^{i+1}}\left(k_{1}^{2}+k_{2}^{2}+k_{3}^{2}\right)^{-1 / 2} \chi(k,-k-n, n) \leq 8+8 \sum_{0<\left|k^{\prime}\right|<2^{i+1}}\left(k_{1}^{2}+k_{2}^{2}\right)^{-1 / 2} \leq C_{0} 2^{i}
$$

where $C_{0}$ is an absolute constant. The first 8 on the right hand side of the above inequality accounts for $k^{\prime}=0$. Therefore, the inequality (4.20) holds with $\alpha=\beta=1$. Let $v_{k}=|k|\left|\mathbf{w}_{A G, k}\right|$ and similarly for $m$ and $n$. Since $\|v\|_{1 / 2}=\left\|\mathbf{w}_{A G}\right\|_{3 / 2},\|v\|_{0}=\left\|\mathbf{w}_{A G}\right\|_{1}$, equations (4.22-4.24) imply

$$
\begin{aligned}
\left|\left(\mathbf{B}_{3}\left(\mathbf{w}_{A G}, \mathbf{w}_{A G}\right),(-\Delta) \mathbf{w}_{A G}\right)\right| & \leq c^{\prime} \sum_{\substack{k+m+n=0\\
}}\left|v_{k}\left\|v_{m}\right\| v_{n}\right| \chi(k, m, n) \\
& \leq c^{\prime} C\|v\|_{1 / 2}^{2}\|v\|_{0}=c^{\prime} C\left\|\mathbf{w}_{A G}\right\|\left\|_{1}\right\| \mathbf{w}_{A G} \|_{3 / 2}^{2} .
\end{aligned}
$$

After that we apply the interpolation inequality $\left\|\mathbf{w}_{A G}\right\|_{3 / 2}^{2} \leq$ const $\left\|\mathbf{w}_{A G}\right\|_{1}\left\|\mathbf{w}_{A G}\right\|_{2}$ and obtain from (4.30) the estimate (4.14) (where the constant depends on $a_{1}, a_{2}, a_{3}$ in general case). This concludes the proof of Theorem 4.1 .

We note that the operator $\mathbf{B}_{3}$ is a bilinear convolution-type operator with the domain of summation $K^{*}$ given by (4.12) (the set of strict 3-waves resonances). The estimate (4.14) for $\mathbf{B}_{3}$ is for the "worst case" of all interactions on the " $2 \frac{1}{2}$-dimensional" interaction manifold $K^{*}$.

\section{INFINITE TIME REGULARITY FOR FINITE LARGE $N$}

In this section we establish global existence and regularity of solutions of equations (2.1) (hence (1.1-1.2)) for $N$ large enough, including the case of all 3-wave resonances, where $\mathbf{B}_{3}\left(\mathbf{w}_{A G}, \mathbf{w}_{A G}\right)$ is present in the limit equation (2.14). The proof of global regularity of 3D "primitive" Navier-Stokes equations (2.1) for resonant domains presented in this paper relies on the global regularity of the " $2 \frac{1}{2}$-dimensional" limit nonlinear NavierStokes equations $(2.14,3.31,3.32)$ and techniques for convergence theorems as $N \rightarrow \infty$ developed in $[3,8,12]$. We impose in our regularity theorems only an integral regularity condition on the forcing term $\mathbf{F}^{\dagger}$ of the type (where $F_{4}$ was rescaled as in Sect. 2)

$$
\sup _{T} \int_{T}^{T+1}\left\|\mathbf{F}^{\dagger}\right\|_{\alpha-1}^{2} \mathrm{~d} t \leq M_{\alpha F}^{2}
$$

where $\alpha>1 / 2$.

In BMN $[5,8,12]$ we proved the regularity of solutions for smooth enough initial data $\mathbf{U}^{\dagger}(0)$ and forcing term $\mathbf{F}^{\dagger}$ for almost all aspect ratios (no strict 3-wave resonances were allowed in these regularity theorems). Now we prove the regularity for all aspect ratios and all 3 -waves resonances. Also, following Avrin and BMN [3] we relax the smoothness conditions on $\mathbf{U}^{\dagger}(0, x)$ and $\mathbf{F}^{\dagger}(t, x)$ using a simple argument based on our previous results on equations with smooth data and using approximation of the data by smooth functions. In fact, we show that we can extend our previous results with very smooth initial data and forcing terms to non-smooth case by continuity. First, we replace $\mathbf{U}^{\dagger}(0)$ and $\mathbf{F}^{\dagger}$ respectively by smooth initial data $\mathbf{U}_{s}^{\dagger}(0) \in H_{\sigma}$ and force $\mathbf{F}_{s}^{\dagger}(t)$ with $\mathbf{F}_{s}^{\dagger}(t) \in H_{\sigma}, \partial_{t} \mathbf{F}_{s}^{\dagger}(t) \in H_{\sigma}, \sigma>\alpha+4$, which are close to $\mathbf{U}^{\dagger}(0)$ and $\mathbf{F}^{\dagger}$. Namely, for approximation of initial data

$$
\left\|\mathbf{U}^{\dagger}(0)-\mathbf{U}_{s}^{\dagger}(0)\right\|_{\alpha} \leq \epsilon .
$$


Further, we assume that $\mathbf{F}^{\dagger}$ is approximated by $\mathbf{F}_{s}^{\dagger}$. We denote $\mathbf{F}^{\prime}=\mathbf{F}^{\dagger}-\mathbf{F}_{s}^{\dagger}$ and assume

$$
\sup _{T} \int_{T}^{T+1}\left\|\mathbf{F}^{\prime}\right\|_{\alpha-1}^{2} \mathrm{~d} t \leq \epsilon^{2}
$$

with $\alpha>1 / 2$.

Of course norms in $H_{\sigma}, \sigma>\alpha+4$ of approximations $\mathbf{U}_{s}^{\dagger}(0), \mathbf{F}_{s}^{\dagger}$ and $\partial_{t} \mathbf{F}_{s}^{\dagger}$ tend to infinity as $\epsilon \rightarrow 0$, but they are bounded for every non-zero $\epsilon$. Using results of [8] we will find a solution $\mathbf{U}_{s}^{\dagger}(t)$ of (2.1) with mollified data which satisfies $\epsilon$-independent estimates in $H_{\alpha}$ for large $N$ (see Theorem 5.2 below). The solutions $\mathbf{U}_{s}^{\dagger}, \mathbf{U}^{\dagger}$ satisfy equations of the form

$$
\begin{gathered}
\partial_{t} \mathbf{U}^{\dagger}(t)=\mathbf{B}\left(\mathbf{U}^{\dagger}, \mathbf{U}^{\dagger}\right)+\overline{\boldsymbol{\nu}} \Delta \mathbf{U}^{\dagger}-N \mathbf{P}^{\dagger} \mathbf{M} \mathbf{P}^{\dagger} \mathbf{U}^{\dagger}+\mathbf{F}^{\dagger} \\
\partial_{t} \mathbf{U}_{s}^{\dagger}(t)=\mathbf{B}\left(\mathbf{U}_{s}^{\dagger}, \mathbf{U}_{s}^{\dagger}\right)+\overline{\boldsymbol{\nu}} \Delta \mathbf{U}_{s}^{\dagger}-N \mathbf{P}^{\dagger} \mathbf{M} \mathbf{P}^{\dagger} \mathbf{U}_{s}^{\dagger}+\mathbf{F}_{s}^{\dagger}
\end{gathered}
$$

with the same bilinear operator $\mathbf{B}$ and different (but close) initial data. In the above equations $\overline{\boldsymbol{\nu}}=$ $\operatorname{diag}\left(\nu_{1}, \nu_{1}, \nu_{1}, \nu_{2}\right)$ is the viscosity matrix, $\mathbf{P}^{\dagger}=(\mathbf{P}, I d)$ where $\mathbf{P}$ is the Leray projection operator. The difference $\Xi(t)=\mathbf{U}^{\dagger}(t)-\mathbf{U}_{s}^{\dagger}(t)$ satisfies the equation

$$
\partial_{t} \Xi(t)=\mathbf{B}\left(\Xi, \mathbf{U}_{s}^{\dagger}\right)+\mathbf{B}\left(\mathbf{U}_{s}^{\dagger}, \Xi\right)+\mathbf{B}(\Xi, \Xi)+\overline{\boldsymbol{\nu}} \Delta \Xi-N \mathbf{P}^{\dagger} \mathbf{M} \mathbf{P}^{\dagger} \Xi+\mathbf{F}^{\prime}, \Xi(0)=\mathbf{U}^{\dagger}(0)-\mathbf{U}_{s}^{\dagger}(0)
$$

with a small forcing term $\mathbf{F}^{\prime}=\mathbf{F}^{\dagger}-\mathbf{F}_{s}^{\dagger}$, and small initial data $\Xi(0)$.

Theorem 5.1. Let $\alpha>3 / 4, \nu_{1}, \nu_{2}>0, T_{0}>0$. Let (5.2-5.3) hold and

$$
\left\|\mathbf{U}_{s}^{\dagger}(t)\right\|_{\alpha} \leq M_{s, \alpha}, 0 \leq t \leq T_{0}, \nu \int_{0}^{T_{0}}\left\|\mathbf{U}_{s}^{\dagger}(t)\right\|_{\alpha+1}^{2} \leq M_{s, \alpha}^{2}
$$

let $\epsilon \leq \epsilon_{0}$, where $\epsilon_{0}$ depends on $M_{s, \alpha}, \alpha, \nu, T_{0}$. Then a regular solution $\Xi$ of equation (5.5) exists and

$$
\|\Xi(t)\|_{\alpha} \leq C_{0} \epsilon, 0 \leq t \leq T_{0} ; \nu \int_{0}^{T_{0}}\|\Xi(t)\|_{\alpha+1}^{2} \leq C_{0}^{2} \epsilon^{2} .
$$

where $C_{0}$ depends on $M_{s, \alpha}, \alpha, \nu ; \nu=\min \left(\nu_{1}, \nu_{2}\right)$.

For the proof of Theorem 5.1 we refer to $[12,14]$.

We now sketch the existence theorem for equation (2.1) with smooth in $H_{\sigma}$ initial data extending the results of [12] to cover 3-wave resonance operator.

Theorem 5.2. Let $\eta=f / N$ and the domain parameters $a_{1}, a_{2}$ and $a_{3}$ be arbitrary and fixed; let $\alpha>3 / 4$, $\nu_{1}, \nu_{2}>0, \sigma>\alpha+4, T_{0}>0$ and let $\mathbf{U}_{s}^{\dagger}(t)$ be a solution of (2.1) with smooth initial data and forcing term such that

$$
\begin{gathered}
\left\|\mathbf{U}_{s}^{\dagger}(0)\right\|_{\alpha} \leq M_{\alpha}, \sup _{T} \int_{T}^{T+1}\left\|\mathbf{F}_{s}^{\dagger}\right\|_{\alpha-1}^{2} \mathrm{~d} t \leq\left(M_{\alpha F}\right)^{2} ; \\
\left\|\mathbf{U}_{s}^{\dagger}(0)\right\|_{\sigma} \leq M_{\sigma F}, \sup _{T} \int_{T}^{T+1}\left\|\mathbf{F}^{\dagger}\right\|_{\sigma-1}^{2} \mathrm{~d} t \leq\left(M_{\sigma F}\right)^{2}, \sup _{T} \int_{T}^{T+1}\left\|\partial_{t} \mathbf{F}_{s}^{\dagger}\right\|_{\sigma}^{2} \mathrm{~d} t \leq\left(M_{\sigma F}\right)^{2} .
\end{gathered}
$$


Then for every $N \geq N_{0}\left(M_{\alpha}, M_{\alpha F}, M_{\sigma F}, \nu, a_{1}, a_{2}, a_{3}\right)$, there exists a unique solution $\mathbf{U}_{s}^{\dagger}(t)$ to equation (2.1) for $0 \leq t \leq T_{0}$ such that

$$
\begin{gathered}
\left\|\mathbf{U}_{s}^{\dagger}(t)\right\|_{\alpha} \leq M_{\alpha}^{\prime}, 0 \leq t \leq T_{0} \\
\nu \int_{0}^{T_{0}}\left\|\mathbf{U}_{s}^{\dagger}(t)\right\|_{\alpha+1}^{2} \mathrm{~d} t \leq\left(M_{\alpha}^{\prime}\right)^{2}
\end{gathered}
$$

where $M_{\alpha}^{\prime}$ depends only on $M_{\alpha}, M_{\alpha F}, \nu, a_{1}, a_{2}, a_{3}, T_{0} ; \nu=\min \left(\nu_{1}, \nu_{2}\right)$.

For the proof we refer to [12] and [14]; note that in [12], the " $2 \frac{1}{2}$-dimensional" case was not included, in contrast to [14].

We now conclude with the existence and regularity theorem for less smooth $\mathbf{U}^{\dagger}(0)$ and $\mathbf{F}^{\dagger}$, by bootstrapping local existence with the help of Theorems 5.1 and 5.2.

Theorem 5.3. Let $\eta=f / N$ and the domain parameters $a_{1}, a_{2}$, $a_{3}$ be fixed but arbitrary. Let $\nu_{1}, \nu_{2}>0$, $\nu=\min \left(\nu_{1}, \nu_{2}\right), \alpha>3 / 4$ and the condition (5.1) on the force $\mathbf{F}^{\dagger}(t, x)$ holds. Let

$$
\left\|\mathbf{U}^{\dagger}(0)\right\|_{\alpha} \leq \tilde{M}_{\alpha}
$$

and $N$ be large: $N \geq N_{1}\left(\tilde{M}_{\alpha}, M_{\alpha F}, \nu, a_{1}, a_{2}, a_{3}\right)$. Then solutions of the $3 D$ rescaled primitive Navier-Stokes system (2.1) (hence (1.1-1.2)) are regular for all $t \geq 0$, and

$$
\left\|\mathbf{U}^{\dagger}(t)\right\|_{\alpha} \leq \tilde{M}_{\alpha}^{\prime} \quad \text { for all } t \geq 0
$$

Proof. First, we have a regular solution $\mathbf{U}^{\dagger}(t) \in H_{\alpha}$ with $\left\|\mathbf{U}^{\dagger}(t)\right\|_{\alpha} \leq M_{\alpha}\left(\tilde{M}_{\alpha}, M_{\alpha F}, \nu\right)$ on a small time interval $\left[0, T_{\alpha}\right], T_{\alpha}=T_{\alpha}\left(\tilde{M}_{\alpha}, M_{\alpha F}, \nu\right)$. We consider the case $3 / 4<\alpha \leq 1$. We have the energy estimate for regular solutions:

$$
\begin{gathered}
\left\|\mathbf{U}^{\dagger}(t)\right\|_{0} \leq M_{0}\left(\tilde{M}_{\alpha}, M_{\alpha F}, \nu\right) \quad \forall t \geq 0 \\
\nu \int_{T}^{T+\tau}\left\|\mathbf{U}^{\dagger}(t)\right\|_{1}^{2} \leq M_{0}^{2} \forall T \geq 0 ; 0 \leq \tau \leq 1,
\end{gathered}
$$

where $\nu=\min \left(\nu_{1}, \nu_{2}\right)$.

Remark 5.1. Uniform boundedness of the energy for the condition (5.1) on $\mathbf{F}^{\dagger}(t, x)$ follows from the usual Gronwall inequality estimate:

$$
\left\|\mathbf{U}^{\dagger}(t)\right\|_{0}^{2} \leq\left\|\mathbf{U}^{\dagger}(0)\right\|_{0}^{2} e^{-\nu \lambda_{1} t}+\frac{1}{\nu \lambda_{1}^{\alpha}} \int_{0}^{t} e^{-\nu \lambda_{1}(t-s)}\left\|\mathbf{F}^{\dagger}(s)\right\|_{\alpha-1}^{2} \mathrm{~d} s,
$$

and

$$
\left\|\mathbf{U}^{\dagger}(t)\right\|_{0}^{2} \leq\left\|\mathbf{U}^{\dagger}(0)\right\|_{0}^{2} e^{-\nu \lambda_{1} t}+\frac{M_{\alpha F}^{2}}{\nu \lambda_{1}^{\alpha}} \frac{1}{1-e^{-\nu \lambda_{1}}}
$$

whence

$$
M_{0}^{2}=C_{\alpha}^{2} \tilde{M}_{\alpha}^{2}+M_{\alpha F}^{2}\left(1+\frac{1}{\nu \lambda_{1}^{\alpha}\left(1-e^{-\nu \lambda_{1}}\right)}\right)
$$


Here $C_{\alpha}$ is embedding constant from $H_{0}$ to $H_{\alpha}$ and $\lambda_{1}$ is the first eigenvalue of the Stokes operator.

For every $t \geq \tau$, equation (5.13) implies that on every interval $[t-\tau, t]$ including $t=\tau$, we have a point $t^{*}$ for which:

$$
\left\|\mathbf{U}^{\dagger}\left(t^{*}\right)\right\|_{1} \leq M_{0} / \sqrt{\nu \tau} .
$$

From now on, we choose $\tau=T_{\alpha}$, local existence time defined above. For every $t \geq T_{\alpha}$, we take $\mathbf{U}^{\dagger}\left(t^{*}\right)$ as new initial data, with $t-T_{\alpha} \leq t^{*} \leq t$. To prove that the solution is uniformly bounded in $H_{\alpha}$ for all $t \geq T_{\alpha}$, it suffices to derive uniform bound for $t \in\left[t^{*}, t^{*}+T_{\alpha}\right]$, with the help of Theorems 5.1 and 5.2; in both theorems we set $T_{0}=T_{\alpha}$. At $t=t^{*}$, the initial condition (5.8) of Theorem 5.2 becomes:

$$
M_{\alpha}=C_{\alpha, 1} M_{0} / \sqrt{\nu T_{\alpha}},
$$

$C_{\alpha, 1}$ embedding constant from $H_{\alpha}$ to $H_{1}$.

Approximating the force $\mathbf{F}^{\dagger}(t), \partial_{t} \mathbf{F}^{\dagger}(t)$ and initial data $\mathbf{U}^{\dagger}\left(t^{*}\right)$ by smooth functions $\mathbf{F}_{s}^{\dagger}(t), \partial_{t} \mathbf{F}_{s}^{\dagger}(t), \mathbf{U}_{s}^{\dagger}\left(t^{*}\right)$ in $H_{\alpha}$ we obtain that

$$
\left\|\Xi\left(t^{*}\right)\right\|_{\alpha}=\left\|\mathbf{U}^{\dagger}\left(t^{*}\right)-\mathbf{U}_{s}^{\dagger}\left(t^{*}\right)\right\|_{\alpha} \leq \epsilon,\left\|\Xi\left(t^{*}\right)\right\|_{\sigma} \leq M_{\epsilon} .
$$

Moreover, the inequality (5.3) holds and, with $\mathbf{F}^{\prime}=\mathbf{F}^{\dagger}-\mathbf{F}_{s}^{\dagger}$ :

$$
\left\|\mathbf{F}^{\prime}(t)\right\|_{\sigma} \leq M_{\epsilon},\left\|\partial_{t} \mathbf{F}^{\prime}(t)\right\|_{\sigma} \leq M_{\epsilon}, t^{*} \leq t \leq t^{*}+T_{\alpha}
$$

where $M_{\epsilon}$ depends on $M_{\alpha}, M_{\alpha F}$ and $\epsilon$ only; of course, $M_{\alpha}$ in (5.17) depends on the original $\tilde{M}_{\alpha}$, and $M_{\alpha F}, \nu, a_{1}$, $a_{2}, a_{3}$. We choose $\epsilon$ so small that we have by Theorem 5.1 (where $M_{\sigma F}$ is replaced by $M_{\alpha F}$ ) a regular solution $\Xi(t)$ on $\left[t^{*}, t^{*}+T_{\alpha}\right]$ which is bounded in $H_{\alpha}$ by 1 when the initial data are in $H_{\alpha}$. After that we consider the Navier-Stokes equations with smooth initial data $\mathbf{U}_{s}^{\dagger}\left(t^{*}\right)$ and force $\mathbf{F}_{s}^{\dagger}$ which satisfy (5.9) for $t^{*} \leq t \leq t^{*}+T_{\alpha}$.

The $H_{\sigma}$-norms of these smooth functions are bounded by (a possibly large) constant $M_{\epsilon}$ depending on this fixed $\epsilon$ and $M_{\alpha}$ (hence $\left.\tilde{M}_{\alpha}\right), M_{\alpha F}$. After that we choose $N \geq N_{1}\left(\epsilon, M_{\alpha}, M_{\alpha F}\right)$ so large that we have (5.10) and (5.11) for solutions $\mathbf{U}_{s}^{\dagger}(t)$ of equations with smooth data. By (5.10) and (5.7) with $C_{0} \epsilon \leq 1$ we have $\left\|\mathbf{U}^{\dagger}(t)\right\|_{\alpha} \leq\left\|\mathbf{U}_{s}^{\dagger}(t)\right\|_{\alpha}+\left\|\mathbf{U}^{\dagger}(t)-\mathbf{U}_{s}^{\dagger}(t)\right\|_{\alpha} \leq M_{\alpha}^{\prime}+1$ with $M_{\alpha}^{\prime}=M_{\alpha}^{\prime}\left(\tilde{M}_{\alpha}, M_{\alpha F}, \nu, a_{1}, a_{2}, a_{3}\right)$. Setting $\tilde{M}_{\alpha}^{\prime}=\max \left(M_{\alpha}, M_{\alpha}^{\prime}+1\right)$ completes the proof of boundedness of $\mathbf{U}^{\dagger}(t)$ in $H_{\alpha}$ for all $t \geq 0$. We also have

$$
\nu \int_{T}^{T+1}\left\|\mathbf{U}^{\dagger}(t)\right\|_{\alpha+1}^{2} \mathrm{~d} t \leq\left(M_{\alpha}^{\prime \prime}\right)^{2}
$$

for every $T \geq 0$ and $3 / 4<\alpha \leq 1$. To extend the above to the case $\alpha>1$ we use uniform-in- $t$ boundedness in $H_{1}$ already proven and then apply the smoothing property for solutions of Navier-Stokes equations (see Theorem 8.2 in [8]) and obtain that the solutions are bounded for $t \geq t_{*}>0$ in $H_{\alpha}, \alpha>1$ and we get the statement of Theorem 5.3 in this case as well. Theorem 5.3 is proven.

Finally, like in [8] we obtain regularity for all large-enough times for weak solutions of the 3D "primitive" Navier-Stokes equations (1.1-1.2) with a force $\mathbf{F}^{\dagger}(t)$. This theorem describes the situation when $N$ is fixed, and large enough (depending only on magnitude of $\mathbf{F}^{\dagger}(t)$ and independent of the initial data). The situation is that of non-smooth and arbitrary large initial data in $H_{0}$. Then weak Leray solutions $\mathbf{U}^{\dagger}(t)$ always exist (with a possible blow-up in $H_{1}$ at some values of $t<t^{*}$, see [20]), here we show that blow-up cannot happen if $t$ is large.

Theorem 5.4. Let $\eta=f / N$ and the domain parameters $a_{1}, a_{2}, a_{3}$ be fixed but arbitrary. Let $\nu_{1}, \nu_{2}>0, \nu=$ $\min \left(\nu_{1}, \nu_{2}\right), \alpha>3 / 4$ and the condition (5.1) on the force be satisfied. Let $\left\|\mathbf{U}^{\dagger}(0)\right\|_{0} \leq M_{0}, \hat{T}=\hat{T}\left(M_{0}, M_{\alpha F}, \nu\right)$. Then for every $N \geq N^{\prime}\left(a_{1}, a_{2}, a_{3}, \nu, M_{\alpha F}\right), N^{\prime}$ independent of $M_{0}$ and for every weak solution $\mathbf{U}^{\dagger}\left(t, x_{1}, x_{2}, x_{3}\right)$ of 
the three-dimensional "primitive" Navier-Stokes equations (1.1-1.2) defined on $[0, \hat{T}]$ which satisfies the classical energy estimates on $[0, \hat{T}]$, the following holds: $\mathbf{U}^{\dagger}\left(t, x_{1}, x_{2}, x_{3}\right)$ can be extended to $0<t<+\infty$ and it is regular for every $t: \hat{T} \leq t<+\infty ; \mathbf{U}^{\dagger}\left(t, x_{1}, x_{2}, x_{3}\right)$ belongs to $H_{\alpha}$ and $\left\|\mathbf{U}^{\dagger}\left(t, x_{1}, x_{2}, x_{3}\right)\right\|_{\alpha} \leq C_{1}\left(a_{1}, a_{2}, a_{3}, M_{\alpha F}, \nu\right)$ for every $t \geq \hat{T}$ where $M_{\alpha F}$ is the $H_{\alpha}$-norm of $\mathbf{F}^{\dagger}$. If $\mathbf{F}^{\dagger}$ is independent of $t$ then there exists a global attractor for the three-dimensional primitive Navier-Stokes equations (1.1-1.2) bounded in $H_{\alpha}$; such an attractor has a finite fractal dimension and attracts every weak Leray solution as $t \rightarrow+\infty$.

Acknowledgements. We would like to thank Professors V.I. Arnold, C. Bardos and C. Foias for the very useful discussions. The work was supported by the AFOSR contract FG9620-99-1-0300 and the Isaac Newton Institute for Mathematical Sciences, Cambridge University.

\section{REFERENCES}

[1] V.I. Arnold, Small denominators. I. Mappings of the circumference onto itself. Amer. Math. Soc. Transl. Ser. 2. 46 (1965) 213-284.

[2] V.I. Arnold and B.A. Khesin, Topological Methods in Hydrodynamics. Appl. Math. Sci. 125 (1997).

[3] J. Avrin, A. Babin, A. Mahalov and B. Nicolaenko, On regularity of solutions of 3D Navier-Stokes equations. Appl. Anal. 71 (1999) 197-214.

[4] A. Babin, A. Mahalov and B. Nicolaenko, Long-time averaged Euler and Navier-Stokes equations for rotating fluids, In Structure and Dynamics of Nonlinear Waves in Fluids, 1994 IUTAM Conference, K. Kirchgässner and A. Mielke Eds, World Scientific (1995) 145-157.

[5] A. Babin, A. Mahalov and B. Nicolaenko, Global splitting, integrability and regularity of 3D Euler and Navier-Stokes equations for uniformly rotating fluids. Europ. J. Mech. B/Fluids 15, No. 3, (1996) 291-300.

[6] A. Babin, A. Mahalov and B. Nicolaenko, Resonances and regularity for Boussinesq equations. Russian J. Math. Phys. 4, No. 4, (1996) 417-428.

[7] A. Babin, A. Mahalov and B. Nicolaenko, Regularity and integrability of rotating shallow-water equations. Proc. Acad. Sci. Paris Ser. 1324 (1997) 593-598.

[8] A. Babin, A. Mahalov and B. Nicolaenko, Global regularity and integrability of 3D Euler and Navier-Stokes equations for uniformly rotating fluids. Asympt. Anal. 15 (1997) 103-150.

[9] A. Babin, A. Mahalov and B. Nicolaenko, Global splitting and regularity of rotating shallow-water equations. Eur. J. Mech., B/Fluids 16, No. 1, (1997) 725-754.

[10] A. Babin, A. Mahalov and B. Nicolaenko, On the nonlinear baroclinic waves and adjustment of pancake dynamics. Theor. and Comp. Fluid Dynamics 11 (1998) 215-235.

[11] A. Babin, A. Mahalov, B. Nicolaenko and Y. Zhou, On the asymptotic regimes and the strongly stratified limit of rotating Boussinesq equations. Theor. and Comp. Fluid Dyn. 9 (1997) 223-251.

[12] A. Babin, A. Mahalov and B. Nicolaenko, On the regularity of three-dimensional rotating Euler-Boussinesq equations. Math. Models Methods Appl. Sci., 9, No. 7 (1999) 1089-1121.

[13] A. Babin, A. Mahalov and B. Nicolaenko, Global regularity of 3D rotating Navier-Stokes equations for resonant domains. Lett. Appl. Math. (to appear).

[14] A. Babin, A. Mahalov and B. Nicolaenko, Global Regularity of 3D Rotating Navier-Stokes Equations for Resonant Domains. Indiana University Mathematics Journal 48, No. 3, (1999) 1133-1176.

[15] A. Babin, A. Mahalov and B. Nicolaenko, Fast singular oscillating limits of stably stratified three-dimensional Euler-Boussinesq equations and ageostrophic wave fronts, to appear in Mathematics of Atmosphere and Ocean Dynamics, Cambridge University Press (1999).

[16] A.V. Babin and M.I. Vishik, Attractors of Evolution Equations, North-Holland, Amsterdam (1992).

[17] C. Bardos and S. Benachour, Domaine d'analycité des solutions de l'équation d'Euler dans un ouvert de $\mathbb{R}^{n}$. Annali della Scuola Normale Superiore di Pisa 4 (1977) 647-687.

[18] P. Bartello, Geostrophic adjustment and inverse cascades in rotating stratified turbulence. J. Atm. Sci. 52, No. 24, (1995) 4410-4428.

[19] A.J. Bourgeois and J.T. Beale, Validity of the quasigeostrophic model for large-scale flow in the atmosphere and the ocean, SIAM J. Math. Anal. 25, No. 4, (1994) 1023-1068.

[20] L. Caffarelli, R. Kohn and L. Nirenberg, Partial regularity of suitable weak solutions of the Navier-Stokes equations. Comm. Pure Appl. Math. 35 (1982) 771-831.

[21] J.-Y. Chemin, A propos d'un probleme de pénalisation de type antisymétrique. Proc. Paris Acad. Sci. 321 (1995) 861-864.

[22] P. Constantin, The Littlewood-Paley spectrum in two-dimensional turbulence, Theor. and Comp. Fluid Dyn. 9, No. 3/4, (1997) 183-191.

[23] P. Constantin and C. Foias, Navier-Stokes Equations, The University of Chicago Press (1988). 
[24] A. Craya, Contribution à l'analyse de la turbulence associée à des vitesses moyennes. P.S.T. Ministère de l'Air 345 (1958).

[25] P.G. Drazin and W.H. Reid, Hydrodynamic Stability, Cambridge University Press (1981).

[26] P.F. Embid and A.J. Majda, Averaging over fast gravity waves for geophysical flows with arbitrary potential vorticity, Comm. Partial Diff. Eqs. 21 (1996) 619-658.

[27] I. Gallagher, Un résultat de stabilité pour les équations des fluides tournants, C.R. Acad. Sci. Paris, Série I (1997) $183-186$.

[28] I. Gallagher, Asymptotics of the solutions of hyperbolic equations with a skew-symmetric perturbation. J. Differential Equations 150 (1998) 363-384.

[29] I. Gallagher, Applications of Schochet's methods to parabolic equations. J. Math. Pures Appl. 77 (1998) 989-1054.

[30] E. Grenier, Rotating fluids and inertial waves. Proc. Acad Sci. Paris Ser. 1321 (1995) 711-714.

[31] J.L. Joly, G. Métivier and J. Rauch, Generic rigorous asymptotic expansions for weakly nonlinear multidimensional oscillatory waves. Duke Math. J. 70 (1993) 373-404.

[32] J.L. Joly, G. Métivier and J. Rauch, Resonant one-dimensional nonlinear geometric optics. J. Funct. Anal. 114 (1993) $106-231$.

[33] J.L. Joly, G. Métivier and J. Rauch, Coherent nonlinear waves and the Wiener algebra. Ann. Inst. Fourier 44 (1994) $167-196$.

[34] J.L. Joly, G. Métivier and J. Rauch, Coherent and focusing multidimensional nonlinear geometric optics. Ann. Scient. E. N. S. Paris 4 (1995) 28, 51-113.

[35] D.A. Jones, A. Mahalov and B. Nicolaenko, A numerical study of an operator splitting method for rotating flows with large ageostrophic initial data. Theor. and Comp. Fluid Dyn. 13, No. 2, (1998) 143-159.

[36] O.A. Ladyzhenskaya, The Mathematical Theory of Viscous Incompressible Flow, 2nd edition, Gordon and Breach, New York (1969).

[37] J.-L. Lions, R. Temam and S. Wang, Geostrophic asymptotics of the primitive equations of the atmosphere. Topological Methods in Nonlinear Analysis 4 (1994) 253-287, special issue dedicated to J. Leray.

[38] J.-L. Lions, R. Temam and S. Wang, A simple global model for the general circulation of the atmosphere. Comm. Pure Appl. Math. 50 (1997) 707-752.

[39] A. Mahalov, S. Leibovich and E.S. Titi, Invariant helical subspaces for the Navier-Stokes Equations. Arch. for Rational Mech. and Anal. 112, No. 3, (1990) 193-222.

[40] A. Mahalov and P.S. Marcus, Long-time averaged rotating shallow-water equations, Proc. of the First Asian Computational Fluid Dynamics Conference, W.H. Hui, Y.-K. Kwok and J.R. Chasnov Eds, vol. 3, Hong Kong University of Science and Technology (1995) 1227-1230.

[41] O. Métais and J.R. Herring, Numerical experiments of freely evolving turbulence in stably stratified fluids. J. Fluid Mech. 202 (1989) 117

[42] J. Pedlosky, Geophysical Fluid Dynamics, 2nd edition, Springer-Verlag (1987).

[43] H. Poincaré, Sur la précession des corps déformables. Bull. Astronomique 27 (1910) 321.

[44] G. Raugel and G. Sell, Navier-Stokes equations on thin 3D domains. I. Global attractors and global regularity of solutions, J. Amer. Math. Soc. 6, No. 3, (1993) 503-568.

[45] S. Schochet, Fast singular limits of hyperbolic PDE's. J. Differential Equations 114 (1994) 476-512.

[46] E.M. Stein, Singular integrals and differentiability properties of functions, Princeton University Press (1970).

[47] S.L. Sobolev, Ob odnoi novoi zadache matematicheskoi fiziki. Izvestiia Akademii Nauk SSSR, Ser. Matematicheskaia. 18, No. 1, (1954) 3-50.

[48] R. Temam, Navier-Stokes Equations: Theory and Numerical Analysis, North-Holland, Amsterdam (1984).

[49] R. Temam, Navier-Stokes Equations and Nonlinear Functional Analysis, SIAM, Philadelphia (1983). 